\title{
DIFFEOMORPHIC MATCHING AND DYNAMIC DEFORMABLE SURFACES IN 3D MEDICAL IMAGING
}

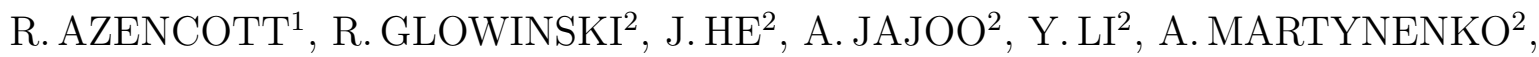 \\ R.H.W.HOPPE ${ }^{3}$, S. BENZEKRY ${ }^{4}$, S.H. LITTLE ${ }^{5}$, AND W.A.ZOGHBI ${ }^{5}$
}

\begin{abstract}
We consider optimal matching of submanifolds such as curves and surfaces by a variational approach based on Hilbert spaces of diffeomorphic transformations. In an abstract setting, the optimal matching is formulated as a minimization problem involving actions of diffeomorphisms on regular Borel measures considered as supporting measures of the reference and the target submanifolds. The objective functional consists of two parts measuring the elastic energy of the dynamically deformed surfaces and the quality of the matching. To make the problem computationally accessible, we use reproducing kernel Hilbert spaces with radial kernels and weighted sums of Dirac measures which gives rise to diffeomorphic point matching and amounts to the solution of a finite dimensional minimization problem. We present a matching algorithm based on the first order necessary optimality conditions which include an initial-value problem for a dynamical system in the trajectories describing the deformation of the surfaces and a final-time problem associated with the adjoint equations. The performance of the algorithm is illustrated by numerical results for examples from medical image analysis.
\end{abstract}

2000 Mathematics Subject Classification: 49M05; 65K10; 68U $0 ; 92 \mathrm{C} 55$.

Keywords: diffeomorphic image matching, deformable surfaces, reproducing kernel Hilbert spaces, Dirac measures, gradient method, medical image analysis.

\section{Introduction}

Clinical diagnosis and therapy planning are increasingly often supported by 3D-imaging modalities, such as MRS (Magnetic Resonance Spectroscopy), PET (Positron Emission Tomography), SPECT (Single Photon Emission Computed Tomography) for functional information, and CT (Computed Tomography), MRI (Magnetic Resonance Imaging), Ultrasound Echography, X-Ray, for anatomical visualization.

Thus clinicians and medical researchers become natural users for automated 3D-image registration providing voxel to voxel matching of two 3D-images of the same anatomical object

\footnotetext{
${ }^{1}$ Department of Mathematics, University of Houston, Houston, TX 77204, U.S.A.; Ecole Normale Superieure, Cachan, F-94005, France. E-mail: razencot@math.uh.edu

${ }^{2}$ Department of Mathematics, University of Houston, Houston, TX 77204, U.S.A. E-mail: roland@math.uh.edu, jiwenhe@math.uh.edu, ajajoo@math.uh.edu, ypli@math.uh.edu, andrey@math.uh.edu

${ }^{3}$ Department of Mathematics, University of Houston, Houston, TX 77204, U.S.A.; Institute of Mathematics, University of Augsburg, D-86159 Augsburg, Germany. E-mail: rohop@math.uh.edu, hoppe@math.uniaugsburg.de

${ }^{4}$ Medical Office, 7500 Kirby Drive, Houston, TX 77030, U.S.A.

${ }^{5}$ The Methodist Hospital Research Institute, Houston, TX 77030, U.S.A. E-mail: wzoghbi@tmhs.org
} 
obtained by different imaging modalities, at different times, or from different perspectives. The search for a good voxel to voxel correspondence between reference and target images $J_{\text {ref }}$ and $J_{\text {tar }}$ is guided by one or several matching quality criteria. Image matching is generally achieved by an $\mathbb{R}^{3}$-diffeomorphism $F$ matching two given bounded subdomains of the $3 \mathrm{D}$-voxel grid, and can thus be assigned an elastic energy $\mathrm{EE}(F)$ measuring the amount of spatial deformation implemented by $F$. In 2D or 3D-image registration, typical matching quality criteria involve the differences $\operatorname{difint}\left(z, z^{\prime}\right)=\left|J_{\text {tar }}\left(z^{\prime}\right)-J_{\text {ref }}(z)\right|$ in image intensities at all pairs $\left(z, z^{\prime}\right)$ of matched voxels. The Intensity Matching Cost $\operatorname{IMC}(F)$ is often defined by fixing some exponent $a>0$ and summing $\operatorname{difint}^{a}\left(z, z^{\prime}\right)$ over all voxels $z$ belonging to the domain of interest in $J_{\text {ref }}$. The search for an optimal registration then becomes a variational problem where one seeks a deformation $F$ minimizing a linear combination of $\operatorname{EE}(F)$ and $\operatorname{IMC}(F)$. Image registration methods were initially designed for 2D-images, for instance to align tomographic slices of different recordings, but in the last decade, 3D-image registration based on volumetric data sets has become the main technical challenge, and involves much heavier computing resources. Surveys of image registration algorithms can be found in $[10,26,37]$.

\section{Diffeomorphic Shape Matching}

\subsection{Diffeomorphic Matching of Two Shapes in $\mathbb{R}^{3}$}

In most medical imaging applications, different 3D-shapes $B \subset \mathbb{R}^{3}$ of the same deformable organ, such as the brain or the heart, can be assumed to belong to the following family SH3 of smooth 3D-shapes with boundaries: We define the family SH3 of 3D-shapes as the set of all connected open subsets $S$ of $\mathbb{R}^{3}$ with compact closures $\bar{S}$ such that

- the boundary $\Sigma=\partial S=\bar{S}-S$ of $S$ is a piecewise smooth surface of class $C_{r}, r \geqslant 3$;

- $S$ coincides with a whole connected component of $\mathbb{R}^{3} \backslash \Sigma$;

- for each $x \in \Sigma$, there is an open neighborhood $U$ of $x$ in $\mathbb{R}^{3}$ and a local $r$-smooth diffeomorphism $\varphi$ of $U$ onto an open ball $\tilde{U} \subset \mathbb{R}^{3}$ mapping $U \cap \bar{\Sigma}$ onto $\tilde{U} \cap H$, where $H$ is the intersection of either one, two or three closed half-spaces of $\mathbb{R}^{3}$.

In medical 3D-imaging, one of the main goals of image registration is to accurately compare two observed 3D-shapes $S_{\text {ref }} \in \mathrm{SH} 3$ and $S_{t a r} \in \mathrm{SH} 3$ of the same deformable organ. These $3 \mathrm{D}$-shapes are often extracted from two $3 \mathrm{D}$-images $J_{\text {ref }}$ and $J_{t a r}$ by various $3 \mathrm{D}$-image analysis techniques such as 3D-segmentation combined with interactive tagging of reference points. We refer to $\Sigma_{r e f}$ and $\Sigma_{t a r}$ as the boundaries of $S_{\text {ref }}$ and $S_{t a r}$. Since these two 3D-shapes are in SH3, any $\mathbb{R}^{3}$-diffeomorphism $F$ such that $F\left(\Sigma_{r e f}\right)=\Sigma_{\text {tar }}$ must necessarily also verify $F\left(S_{r e f}\right)=S_{t a r}$. Hence, in the search for a diffeomorphic matching of two deformable 3Dshapes, whenever theses shapes have been already pre-extracted as $\mathbb{R}^{3}$-subsets, the matching of image intensities is then often discarded, and the matching quality of candidate $\mathbb{R}^{3}$ diffeomorphisms $F$ is focused on suitable geometric distances between the boundary surfaces $F\left(\Sigma_{r e f}\right)$ and $\Sigma_{t a r}$ as well as between the 3D-shapes $F\left(S_{r e f}\right)$ and $S_{t a r}$.

As just indicated, diffeomorphic matching of 3D-shapes $S_{0}, S_{1}$ in $\mathbb{R}^{3}$ quite naturally involves looking at the matching of their boundaries $\Sigma_{0}, \Sigma_{1}$ which are 2D-shapes in $\mathbb{R}^{3}$ as well as to the matching in $\mathbb{R}^{3}$ of the boundary 1 D-shapes $\partial \Sigma_{0}, \partial \Sigma_{1}$, where the definitions of smooth $2 \mathrm{D}$ and $1 \mathrm{D}$-shapes in $\mathbb{R}^{3}$ are similar to the definition of SH3. 


\subsection{Variational Approach}

Consider two smooth k-dimensional shapes $S_{0}, S_{1}$ included in $\mathbb{R}^{3}$ with $k \in\{1,2,3\}$. The search for an $\mathbb{R}^{3}$-diffeomorphism $F$ such that $F\left(S_{0}\right)=S_{1}$ is an ill-posed problem which requires regularization to be numerically solved by variational methods. Such an approach has been actively explored by G.Dupuis, J. Glaunès, U. Grenander, M. Miller, D. Mumford, A. Trouvé, L. Younes $[9,16,21,22]$ with applications to a quantified comparison of images of human brains. In these papers, the regularization is achieved through the replacement of the rigid constraint $F\left(S_{0}\right)=S_{1}$ by a soft constraint based on various geometric 'surface matching' distances dis $\left[F\left(S_{0}\right), S_{1}\right]$. The unknown diffeomorphism $F$ is restricted to be of the form $F=F^{v}$, where $F^{v}$ is generated by integration between times 0 and 1 of some time dependent flow $v=\left(v_{t}\right)$ of smooth $\mathbb{R}^{3}$-vector fields $v_{t}, 0 \leqslant t \leqslant 1$. The vector fields $v_{t}$ are required to belong to a Hilbert subspace $V$ of the Banach space $C_{r}^{3}$ of smooth functions from $\mathbb{R}^{3}$ to $\mathbb{R}^{3}$ tending to zero at infinity. The Hilbert norm in $V$ is assumed to be bounded by a constant multiple of the Banach norm in $C_{r}^{3}$. Then, for some fixed constant $\lambda>0$ one considers the variational problem of finding a vector field flow $v=\left(v_{t}\right)$ minimizing the cost functional

$$
J(v)=\int_{0}^{1}\left\|v_{t}\right\|_{V}^{2} d t+\lambda \operatorname{dis}\left[F^{v}\left(S_{0}\right), S_{1}\right]
$$

which linearly combines a kinetic energy term and a surface matching term.

This variational point of view is directly linked (as $\lambda \rightarrow \infty)$ to the construction of geodesics in infinite dimensional Lie groups of diffeomorphisms in the spirit of ideas pioneered by Arnold, Ebin, and Marsden who showed (see, e.g., [3]) that for an incompressible fluid, obeying Euler equations, the spatial displacements $F_{t}(x)$ between times 0 and $t$ of fluid particles emanating from $x \in \mathbb{R}^{3}$ minimize the integral in time and space of the fluid kinetic energy. The time dependent $\mathbb{R}^{3}$ - diffeomorphisms $F_{t}$ define a continuous path in the group of $\mathbb{R}^{3}$ - diffeomorphisms, and this path is a geodesic $t \rightarrow F_{t}$ of an infinite dimensional Lie group $G$ of $\mathbb{R}^{3}$-diffeomorphisms, endowed with the local Hilbert metric defined by the fluid's kinetic energy on the Lie algebra of $G$. This Lie algebra is naturally identified with the Hilbert space of smooth vector fields on $\mathbb{R}^{3}$ defined by fluid velocities at time 0 . The classical Euler fluid mechanical equations for the fluid velocities become precisely interpreted as the variational equations characterizing geodesics in $G$. Natural right-invariant deformation distances on the group $G$ can then be associated to this Riemannian structure (see, e.g., [35]).

For the diffeomorphic matching of two smooth $k$-dimensional shapes $(k \in\{1,2,3\})$ by $\mathbb{R}^{3}$ diffeomorphisms, the geometric view just outlined above has been intensively explored in $[9,16,21,35]$ and numerically implemented for comparisons of key anatomic parts of human brains such as the hippocampus, the temporal lobes, etc. [9, 21].

\subsection{Multiple Snapshots of Dynamic Deformable Shapes}

In medical domains such as cardiology, urology, gynecology, clinicians routinely use volumetric 3D-echographs to visualize live 3D-movies of deformable organs. However, computerized algorithms to model such soft organs dynamics by explicit time dependent nonlinear 3Ddeformations constitute a quite complex and active research target. For deformable anatomic shapes $S(t) \subset \mathbb{R}^{3}$ indexed by time $t$, current bio-medical research often succeeds to extract from medical 3D-movie data at key time frames $t_{j}, 0 \leqslant j \leqslant q$, a sequence of static models $S_{j} \subset \mathbb{R}^{3}$ of the shapes $S\left(t_{j}\right)$. Given these $q+1$ shape snapshots $S_{j}=S\left(t_{j}\right) \subset \mathbb{R}^{3}, 0 \leqslant j \leqslant q$, 
a natural goal is to model the time deformations of the shape $S(t)$ by a time dependent family $F_{t}$ of $\mathbb{R}^{3}$-diffeomorphisms such that

$$
\begin{gathered}
F_{t_{j}}\left(S_{0}\right)=S_{j} \text { for } j=1, \cdots, q, \\
F_{t_{0}}=I d \text { (identity mapping of } \mathbb{R}^{3} \text { ), } \\
\text { for each fixed t }\left[F_{t}(x)-x\right] \rightarrow 0 \text { as } x \rightarrow \infty \text { in } \mathbb{R}^{3} .
\end{gathered}
$$

Most publications mentioned above deal with the basic case $q=1$ where one wants to match, by an $\mathbb{R}^{3}$-diffeomorphism, a single pair of static smooth $k$-dimensional shapes called reference and target shapes.

In this paper, we will focus on the situation where we are given $q+1$ 'snapshots' $S_{j} \subset$ $\mathbb{R}^{3}$ which is a natural context for $3 \mathrm{D}$-movies analysis. In particular, we will extend the variational approach described above to the search of time dependent $\mathbb{R}^{3}$-diffeomorphisms $F_{t}$ verifying at fixed intermediary times $t_{j}$ the $q>1$ geometric matching constraints listed in (2.1a)-(2.1c). The $q+1$ given 'snapshots' $S_{j} \subset \mathbb{R}^{3}$ are typically smooth $3 \mathrm{D}$-shapes belonging to SH3 (see subsection 2.1) or piecewise smooth $\mathbb{R}^{3}$-submanifolds of lower dimension $k \in$ $\{1,2\}$.

As a medical application we will present a dynamic sequence of mitral valve snapshots where each snapshot $S_{j}$ actually belongs to a more general class of composite deformable objects $S_{j}$ which are unions of several bounded piecewise smooth surfaces and curves in $\mathbb{R}^{3}$ linked by flexible articulations.

\section{Optimal Diffeomorphic Matching of Intermediary Snapshots}

In this section, we will use standard notation from Lebesgue and Sobolev space theory (cf., e.g., [34]). Moreover, $C$ will denote a generic positive constant not necessarily the same at each occurrence.

\subsection{Time Dependent Vector Fields with Finite Kinetic Energy}

We choose a Hilbert space $V$ of vector fields on $\mathbb{R}^{3}$ and we consider the associated Hilbert space $L^{2}(I, V)$ of vector field flows $v: t \rightarrow v_{t} \in V$, indexed by a time parameter $t$ in the interval $I=\left[t_{0}, t_{1}\right]$, having finite kinetic energy $E(v)$ defined by

$$
E(v):=\frac{1}{2}\|v\|_{L^{2}(I, V)}^{2}=\frac{1}{2} \int_{t_{0}}^{t_{1}}\left\|v_{t}\right\|_{V}^{2} d t .
$$

We assume that the Hilbert space $V$ of $\mathbb{R}^{3}$-vector fields is continuously embedded in a Sobolev space $W^{s, 2}\left(\mathbb{R}^{3}\right)^{3}$ for some $s>5 / 2$. By the Sobolev embedding theorem, $W^{s, 2}\left(\mathbb{R}^{3}\right)^{3}, s>5 / 2$, is continuously embedded in the Banach space $C^{0,1}\left(\mathbb{R}^{3}\right)^{3}$ of $\mathbb{R}^{3}$-vector fields. We note that in this situation, for each $x \in \mathbb{R}^{3}$, the evaluation map $w \rightarrow w(x)$ from $V$ to $\mathbb{R}^{3}$ is continuous with respect to both the strong and the weak topology on $V$. 


\subsection{Dynamic System of Diffeomorphic Deformations}

For $t \in I$ and $v=\left(v_{t}\right)$ as above, we define the flow of $\mathbb{R}^{3}$-diffeomorphisms $F_{t}$ as the solution of the flow dynamics equations

$$
\begin{aligned}
\partial_{t} F_{t} & =v_{t}\left(F_{t}\right) \quad, \quad t \in I, \\
F_{0} & =\mathrm{Id},
\end{aligned}
$$

where Id refers to the identity map of $\mathbb{R}^{3}$.

Theorem 3.1. Assume $v \in L^{2}(I ; V)$ where $V$ is continuously embedded in $W^{s, 2}\left(\mathbb{R}^{3}\right)$ for some $s>5 / 2$. Then, the initial-value problem (3.2a),(3.2b) admits a unique solution $F_{t}$ with each $F_{t}$ being an $\mathbb{R}^{3}$-diffeomorphism of smoothness class $1 \leqslant r<s-3 / 2$.

Proof. We refer to [20].

\subsection{Self-Reproducing Hilbert Spaces}

Recall that a symmetric real valued kernel $K\left(x, x^{\prime}\right)$ defined for $\left(x, x^{\prime}\right) \in \mathbb{R}^{3} \times \mathbb{R}^{3}$ is called positive definite if for arbitrary vectors $x_{n} \in \mathbb{R}^{3}, n=1, \cdots, N$, the $(N \times N)$ symmetric matrix $K\left(x_{m}, x_{n}\right)$ is positive definite.

To any such kernel $K$, one associates the vector space LW of all finite linear combinations of $\mathbb{R}^{3}$-vector fields $w_{z, u}$, indexed by arbitrary pairs $(z, u)$ in $\mathbb{R}^{3} \times \mathbb{R}^{3}$, and defined by $w_{z, u}(x)=$ $K(z, x) u$ for all $x \in \mathbb{R}^{3}$. The space $L W$ is then endowed with the pre-Hilbertian scalar product

$$
\left\langle w_{z, u}, w_{z^{\prime}, u^{\prime}}\right\rangle=K\left(z, z^{\prime}\right)\left\langle u, u^{\prime}\right\rangle_{\mathbb{R}^{3}}
$$

The self-reproducing Hilbert space $V_{K}$ classically defined by $K$ is then the unique Hilbert space generated by LW (see $[4,33]$ ).

In our context, the relevant Hilbert space $V$ of $\mathbb{R}^{3}$-vector fields is often defined as the self-reproducing Hilbert space $V=V_{K}$ of $\mathbb{R}^{3}$-vector fields defined by a smooth symmetric bounded positive definite kernel $K$ on $\mathbb{R}^{3} \times \mathbb{R}^{3}$, where $K$ is assumed to be bounded, smooth, and invariant under translations.

For many shape matching applications, $K$ can be the radial Gaussian kernel $K_{\sigma}$

$$
K_{\sigma}\left(x, x^{\prime}\right)=\frac{1}{(2 \pi)^{3 / 2} \sigma^{3}} \exp \left(-\frac{\left\|x-x^{\prime}\right\|^{2}}{\sigma^{2}}\right)
$$

with a suitable scale parameter $\sigma>0$. Note that when $V=V_{K_{\sigma}}$, the Sobolev embedding hypothesis above is satisfied for any $s>5 / 2$. The choice $V=V_{K_{\sigma}}$ seems to be a good pragmatic choice for diffeomorphic shape matching applications as seen in previous studies and in our numerical implementations below.

\subsection{Matching Quality: Distances between Shapes}

We consider a given sequence of instantaneous 'shape snapshots' $S_{j}=S_{t_{j}} \subset \mathbb{R}^{3}$ generated by a deformable shape $S_{t}$ at instants $t_{j}, 0 \leqslant j \leqslant q$. Typically, each $S_{j}$ is a $k$-dimensional submanifold $(k \in\{1,2,3\})$ with boundary, regularly embedded in $\mathbb{R}^{3}$. Let $\left(F_{t}\right)$ be a candidate family of $\mathbb{R}^{3}$-diffeomorphisms indexed by time. To compare each given snapshot $S_{j}$ with the 
deformed initial shape $F_{t_{j}}\left(S_{0}\right)$, a key choice is to define smooth non-negative geometric distances $D\left(S, S^{\prime}\right)$ quantifying the geometric disparity between pairs of shapes $S, S^{\prime}$ in $\mathbb{R}^{3}$. The classical Hausdorff disparities $h\left(S, S^{\prime}\right)$ and $h\left(S^{\prime}, S\right)$ between subsets $S, S^{\prime}$ of $\mathbb{R}^{3}$ are defined by

$$
h\left(S, S^{\prime}\right)=\max _{x \in S}\left(\min _{x^{\prime} \in S^{\prime}}\left\|x-x^{\prime}\right\|\right) .
$$

They determine the Hausdorff distance $D_{h}$ by

$$
\left.D_{h}\left(S, S^{\prime}\right)=\max \left(h\left(S, S^{\prime}\right), h\left(S^{\prime}, S\right)\right)\right) .
$$

Hausdorff distances introduce theoretical complications in the variational framework below, since $D_{h}\left(S, S^{\prime}\right)$ is not always smooth with respect to small perturbations of $S$ or of $S^{\prime}$, but Hausdorff disparities are nevertheless quite useful in numerical schemes as will be clarified in the applications below. For many shape matching applications, one can identify as in [21], each submanifold $S$ regularly embedded in $\mathbb{R}^{3}$, with the measure $\mu_{S} \in$ BM3 induced on $S$ by the Lebesgue measure of $\mathbb{R}^{3}$. Here, BM3 is the space of bounded Borel measures $m, m^{\prime}$ on $R^{3}$, endowed with the Hilbert norm $\|m\|_{\Gamma}$ associated with the scalar product

$$
\left\langle m, m^{\prime}\right\rangle_{\Gamma}=\int_{\mathbb{R}^{3}} \int_{\mathbb{R}^{3}} \Gamma\left(x, x^{\prime}\right) d m(x) d m^{\prime}\left(x^{\prime}\right),
$$

where $\Gamma$ is any smooth, symmetric, translation invariant, and bounded positive definite kernel on $\mathbb{R}^{3} \times \mathbb{R}^{3}$, such as the often used radial Gaussian kernel $K_{\sigma}$. The corresponding distance between two bounded Borel subsets $S, S^{\prime}$ of $\mathbb{R}^{3}$ is then defined by

$$
D_{\Gamma}^{2}\left(S, S^{\prime}\right)=\left\|\mu_{S}-\mu_{S^{\prime}}\right\|_{\Gamma}^{2}
$$

and has nice smoothness properties.

Denote by $\operatorname{Diff}\left(\mathbb{R}^{3}\right)$ the space of all $\mathbb{R}^{3}$-diffeomorphisms endowed with the topology of uniform convergence on bounded subsets of $\mathbb{R}^{3}$. Then for any fixed pair $S, S^{\prime}$ of bounded submanifolds regularly embedded in $\mathbb{R}^{3}$, the distance $D_{\Gamma}\left(G(S), S^{\prime}\right)$ is a continuous function of $G \in \operatorname{Diff}\left(\mathbb{R}^{3}\right)$ (see [21]). In applications, for each snapshot $S_{j}=S_{t_{j}}$ one can often identify a well defined set of $p$ reference points

$$
Z_{j}=\left\{z_{j, 1}, \ldots z_{j, p}\right\}
$$

such that the $Z_{j}$ are point-to-point matched by the unknown diffeomorphisms $F_{t}$. To force the diffeomorphic matching of these reference point sets, we will use the obvious pointwise disparity functions

$$
\operatorname{pointdisp}(V)=\sum_{p=1}^{r}\left|F_{t_{j}}\left(z_{0, p}\right)-z_{j, p}\right|^{2} .
$$

Other efficient disparity functions $D\left(S, S^{\prime}\right)$ based on Hilbertian distances between differentiable currents carried by $S$ and $S^{\prime}$ have been introduced in [21]. We now present the variational formulation for diffeomorphic matching of intermediary snapshots. As above, consider a given sequence of $q+1$ instantaneous 'snapshots' $S_{j}=S_{t_{j}} \subset \mathbb{R}^{3}$ generated at fixed intermediary times $t_{j}, 0 \leqslant j \leqslant q$, by a deformable shape $S_{t}$ with unknown dynamics. Each $S_{j}$ is typically a bounded piecewise smooth submanifold with boundary, regularly 
embedded in $\mathbb{R}^{3}$. We seek a vector field flow $v=\left(v_{t}\right)$ on $\mathbb{R}^{3}$ belonging to the Hilbert space $L^{2}(I, V), I:=\left[t_{0}, t_{q}\right]$ such that for $j=1, \cdots, q$, the $\mathbb{R}^{3}$-diffeomorphism $F_{t}$ solution of (3.2a),(3.2b) deforms the initial snapshot $S_{0}$ into a submanifold $\hat{S}_{j}=F_{t_{j}}\left(S_{0}\right)$ 'coinciding' as well as possible with the given snapshot $S_{j}$. We select a disparity functional $D^{2}\left(S, S^{\prime}\right)$ such as one of the functionals introduced above, and quantify the constraint matching adequacy of $v$ by the $q$ numerical disparities

$$
\operatorname{Disp}_{j}(v)=D^{2}\left(\hat{S}_{j}, S_{j}\right) \quad, \quad j=1, \cdots, q .
$$

We fix $q$ positive numerical weights $\lambda_{j}>0$, and we define the disparity cost functional by

$$
\operatorname{Disp}(v):=\sum_{j=1}^{q} \lambda_{j} \operatorname{Disp}_{j}(v) .
$$

These disparity functionals actually belong to a much wider class of functionals $\mathcal{D}(V)$ which we now introduce.

For $v \in L^{2}(I, V)$ we denote by $F_{t}^{v} \in \operatorname{Diff}\left(\mathbb{R}^{3}\right)$ the solution of (3.2a),(3.2b) determined by $v$. We define $\mathcal{D}(V)$ as the space of all disparity functionals Disp $: L^{2}(I, V) \rightarrow \mathbb{R}_{+}$which are of the form

$$
\operatorname{Disp}(v)=\phi\left(F_{t_{1}}^{v}, \ldots, F_{t_{q}}^{v}\right)
$$

for some fixed, but arbitrary choices of the integer $q$, of the instants $t_{1}, \cdots, t_{q}$ in $\mathbb{R}_{+}$, and of the continuous function $\phi:\left(\operatorname{Diff}\left(\mathbb{R}^{3}\right)\right)^{q} \rightarrow \mathbb{R}_{+}$.

\subsection{Existence of a Minimizing Diffeomorphic Flow}

We define the objective functional $J: L^{2}(I, V) \rightarrow \mathbb{R}$ by

$$
J(v):=E(v)+\operatorname{Disp}(v) \quad, \quad v \in L^{2}(I, V),
$$

where $E(v)$ is the kinetic energy as given by (3.1) and $\operatorname{Disp}(v)$ is as in (3.8). We consider the minimization problem

$$
\inf _{v \in L^{2}(I, V)} J(v)
$$

The next result proves the existence of a minimizing diffeomorphic flow solution of (3.10).

Theorem 3.2. The minimization problem (3.10) has a solution $v^{*} \in L^{2}(I, V)$.

Proof. Let $\left\{v^{n}\right\}_{\mathbb{N}}, v^{n} \in L^{2}(I, V), n \in \mathbb{N}$, be a minimizing sequence, i.e.,

$$
J\left(v^{n}\right) \rightarrow \inf _{v \in L^{2}(I, V)} J(v) \quad(n \rightarrow \infty)
$$

Obviously, this sequence is bounded in $L^{2}(I, V)$ and hence is weakly compact. Consequently, we find $v^{*} \in L^{2}(I, V)$ and a subsequence (still indexed by $\mathbb{N}$ ) converging weakly to $v^{*}$ in $L^{2}(I, V)$. This implies

$$
\liminf _{n \rightarrow \infty}\left\|v^{n}\right\|_{L^{2}(I, V)}^{2} \leqslant\left\|v^{*}\right\|_{L^{2}(I, V)}^{2}
$$


We denote by $F_{t}^{n}$ and $F_{t}^{*}$ the unique flows of $\mathbb{R}^{3}$-diffeomorphisms solving (3.2a),(3.2b) with respect to the vector field flows $v^{n}$ and $v^{*}$, respectively. The main part of the proof will be to verify the following key convergence result

$$
F_{t}^{*}(x)=\lim _{n \rightarrow \infty} F_{t}^{n}(x) \quad \forall t \in I, x \in \mathbb{R}^{3},
$$

where, for each fixed $t \in I$, the convergence in (3.13) is uniform in $x$ on bounded subsets $B$ of $\mathbb{R}^{3}$. If we assume that (3.13) holds true, then $F_{t_{j}}^{n} \rightarrow F_{t_{j}}^{*}, 0 \leqslant j \leqslant q$, in $\left.C\left(\mathbb{R}^{3}, \mathbb{R}^{3}\right)\right)$ as $n \rightarrow \infty$ and hence, the continuity hypothesis on the disparity functional implies

$$
\operatorname{Disp}\left(v^{*}\right)=\lim _{n \rightarrow \infty} \operatorname{Disp}\left(v^{n}\right) .
$$

In view of (3.11), (3.12) and (3.14), we obtain

$$
\inf _{v \in L^{2}(I, V)} J(v)=\liminf _{n \rightarrow \infty} J\left(v^{n}\right) \leqslant \liminf _{n \rightarrow \infty} E\left(v^{n}\right)+\lim _{n \rightarrow \infty} \operatorname{Disp}\left(v^{n}\right) \leqslant E\left(v^{*}\right)+\operatorname{Disp}(v *)=J(v *),
$$

which implies

$$
J\left(v^{*}\right)=\inf _{v \in L^{2}(I, V)} J(v)
$$

i.e., $v^{*}$ is a minimizer of $J$.

We now prove the key point (3.13). Let $B$ be a fixed, but arbitrary bounded subset of $\mathbb{R}^{3}$. Since $V$ is continuously embedded in the Banach space of bounded continuous vector fields, we have

$$
\left\|v_{t}(x)\right\|_{\mathbb{R}^{3}} \leqslant C\left\|v_{t}\right\|_{V} \quad \forall x \in \mathbb{R}^{3}, t \in I, v \in V .
$$

Then, the equations (3.2a),(3.2b) imply that for all $(t, x) \in I \times B$ and all $n \in \mathbb{N}$ there holds

$$
\left\|F_{t}^{n}(x)\right\|_{\mathbb{R}^{3}} \leqslant\|x\|_{\mathbb{R}^{3}}+C \int_{t_{0}}^{t}\left\|v_{t}^{n}\right\|_{V} d t \leqslant C\left(1+\left\|v^{n}\right\|_{L^{2}(I, V)}\right) \leqslant C .
$$

Moreover, for all $a, b \in I$ and $x \in \mathbb{R}^{3}$ we have

$$
\begin{aligned}
\left|F_{a}^{n}(x)-F_{b}^{n}(x)\right| & =\left|\int_{a}^{b} v_{t}^{n}\left(F_{t}^{n}(x)\right) d t\right| \leqslant \int_{a}^{b}\left\|v_{t}^{n}\left(F_{t}^{n}(x)\right)\right\|_{\mathbb{R}^{3}} d t \\
& \leqslant C \int_{a}^{b}\left\|v_{t}^{n}\right\|_{V} d t \leqslant C(b-a)^{1 / 2}\left\|v^{n}\right\|_{L^{2}(I, V)} \leqslant C(b-a)^{1 / 2} .
\end{aligned}
$$

The continuous embedding of $V$ into $W^{s, 2}\left(\mathbb{R}^{3}\right)^{3}, s>5 / 2$, yields

$$
\left\|v_{t}(x)-v_{t}(y)\right\| \leqslant c t e\left\|v_{t}\right\|_{V}\|x-y\| \quad \forall x, y \in \mathbb{R}^{3}, \quad t \in I, \quad v \in L^{2}(I, V)
$$

The inequalities (3.16) and (3.17) imply equicontinuity of the functions

$$
(t, x) \in I \times \mathbb{R}^{3} \rightarrow F_{t}^{n}(x) .
$$


Moreover, for $(t, x) \in I \times B$, the sequence of $\mathbb{R}^{3}$-norms $\left\|F_{t}^{n}(x)\right\|$ is bounded. By Ascoli's theorem, after extraction of a subsequence (for ease of notation still denoted $\left\{v^{n}\right\}_{\mathbb{N}}$ ), we may assume that the sequence $\left\{F_{t}^{n}(x)\right\}_{\mathbb{N}}$ converges uniformly for $(t, x) \in I \times B$ to some continuous function $(t, x) \rightarrow u_{t}(x) \in \mathbb{R}^{3}$. Applying this result to a sequence of balls $B \subset \mathbb{R}^{3}$ with fixed center and radii tending to infinity, and selecting a 'diagonal' subsequence of $\left\{v^{n}\right\}_{\mathbb{N}}$, we deduce the existence of a minimizing sequence (still denoted $\left\{v^{n}\right\}_{\mathbb{N}}$ ) and of a continuous function $(t, x) \rightarrow z_{t}^{*}(x) \in \mathbb{R}^{3}$ such that

$$
z_{t}^{*}(x)=\lim _{n \rightarrow \infty} F_{t}^{n}(x) \quad \text { uniformly for }(t, x) \text { in bounded subsets of } I \times \mathbb{R}^{3} \text {. }
$$

We fix a bounded set $B \subset \mathbb{R}^{3}$ and some $\varepsilon>0$. Then, there exits $n_{1} \in \mathbb{N}$ such that for $n>n_{1}$

$$
\left\|F_{t}^{n}(x)-z_{t}^{*}(x)\right\| \leqslant \varepsilon \quad \forall(t, x) \in I \times B .
$$

We choose $\delta>0$ small enough such that the continuous function $z^{*}:(t, x) \rightarrow z_{t}^{*}(x)$ has oscillations less than $\varepsilon$ on any rectangular box in $I \times B$ with diameter less than $\delta$. We further consider a covering of the bounded set $B \subset \mathbb{R}^{3}$ by a finite family $\Omega$ of disjoint rectangular boxes $\omega \in \Omega$ and a finite partition $\Theta$ of the interval $I$ into subintervals $T \in \Theta$ such that all rectangular boxes $T \times \omega$ have diameters less than $\delta$. Clearly, we can then select a function

$$
Z:(t, x) \in I \times \mathbb{R}^{3} \rightarrow Z_{t}(x) \in \mathbb{R}^{3}
$$

which satisfies $Z_{t}(x) \leqslant y(T, \omega)$ on each $T \times \omega$ with $T \in \Theta, \omega \in \Omega$, and such that

$$
\left\|Z_{t}(x)-z_{t}^{*}(x)\right\|<\varepsilon \quad \forall(t, x) \in I \times B .
$$

For $a, b \in I$ and $x \in B$ we want to estimate the integral

$$
\int_{a}^{b}\left(v_{t}^{n}\left(F_{t}^{n}(x)\right)-v_{t}^{*}\left(z_{t}^{*}(x)\right)\right) d t
$$

where the integrand $U:=v_{t}^{n}\left(F_{t}^{n}(x)\right)-v_{t}^{*}\left(z_{t}^{*}(x)\right)$ can be split according to

$$
U=U_{1}+U_{2}+U_{3}+U_{4}
$$

with

$$
\begin{array}{cc}
U_{1}=v_{t}^{n}\left(F_{t}^{n}(x)\right)-v_{t}^{n}\left(z_{t}^{*}(x)\right) \quad, \quad U_{2}=v_{t}^{n}\left(z_{t}^{*}(x)\right)-v_{t}^{n}\left(Z_{t}(x)\right), \\
U_{3}=v_{t}^{*}\left(Z_{t}(x)\right)-v_{t}^{*}\left(z_{t}^{*}(x)\right) \quad, \quad U_{4}=v_{t}^{n}\left(Z_{t}(x)\right)-v_{t}^{*}\left(Z_{t}(x)\right) .
\end{array}
$$

For any two functions $f_{t}(x)$ and $g_{t}(x)$ that are continuous on $I \times B$ with values in $\mathbb{R}^{3}$ and satisfy

$$
\left\|f_{t}(x)-g_{t}(x)\right\|_{\mathbb{R}^{3}} \leqslant \varepsilon \quad \forall(t, x) \in I \times B
$$

we have the bound

$$
\left\|v_{t}\left(f_{t}(x)\right)-v_{t}\left(g_{t}(x)\right)\right\|_{\mathbb{R}^{3}} \leqslant C \varepsilon\left\|v_{t}\right\|_{V} \quad \forall t \in I, \quad x \in B, v \in L^{2}(I, V) .
$$


This implies that for all $a, b \in I, x \in B$ there holds

$$
\left|\int_{a}^{b}\left(v_{t}\left(f_{t}(x)\right)-v_{t}\left(g_{t}(x)\right)\right) d t\right| \leqslant C \varepsilon \int_{a}^{b}\left\|v_{t}\right\|_{V} d t \leqslant C \varepsilon\|v\|_{L^{2}(I, V)} .
$$

Applying the preceding argument separately to $U_{1}, U_{2}, U_{3}$, we find that for all $a, b \in I, x \in B$, and all $n>n_{1}$ we have

$$
\int_{a}^{b}\left[\left|U_{1}\right|+\left|U_{2}\right|+\left|U_{3}\right|\right] d t \leqslant C \varepsilon\left(2\left\|v^{n}\right\|_{L^{2}(I, V)}+\left\|v^{*}\right\|_{L^{2}(I, V)}\right) \leqslant C \varepsilon .
$$

Since the Hilbert space $V$ is continuously embedded in $W^{s, 2}\left(\mathbb{R}^{3}\right)^{3}, s>5 / 2$, for each $y \in \mathbb{R}^{3}$ there exists a function $\operatorname{ker}^{y} \in V$ generating the evaluation map on $V$ by means of

$$
w(y)=\left\langle k e r^{y}, v\right\rangle, \quad \forall w \in V .
$$

Now, for each $y \in \mathbb{R}^{3}$ and each pair $a, b \in I$ we define a function

$$
\mathrm{KER}: t \rightarrow \mathrm{KER}_{t}=1_{[a, b]}(t) \operatorname{ker}^{y}
$$

Clearly, KER $\in L^{2}(I, V)$ and we have

$$
\int_{[a, b]} v_{t}(y) d t=\int_{I}\left\langle\mathrm{KER}, v_{t}\right\rangle_{V} d t=\langle\mathrm{KER}, v\rangle_{V} \quad \forall v \in L^{2}(I, V), y \in \mathbb{R}^{3} .
$$

Since $v^{n}-v^{*} \rightarrow 0$ in $L^{2}(I, V)$, the last equality shows that for each fixed $y \in \mathbb{R}^{3}$ and each pair $a, b \in I$ we have

$$
\lim _{n \rightarrow \infty}\left(\int_{[a, b]}\left(v_{t}^{n}(y)-v_{t}^{*}(y)\right) d t\right)=0 .
$$

For each pair $(T, \omega) \in \Theta \times \Omega$ there is a fixed vector $y(T, \omega) \in \mathbb{R}^{3}$ such that $Z_{t}(x)=y(T, \omega)$ for all $(t, x) \in T \times \omega$. Fixing $\omega \in \Omega$, for all $x \in \omega$ we have

$$
\begin{aligned}
\int_{a}^{b} U_{4} d t & =\sum_{T \in \Theta} \int_{T \cap[a, b]}\left(v_{t}^{n}\left(Z_{t}(x)\right)-v_{t}^{*}\left(Z_{t}(x)\right)\right) d t \\
& =\sum_{T \in \Theta} \int_{T \cap[a, b]}\left(v_{t}^{n}(y(T, \omega))-v_{t}^{*}(t(T, \omega))\right) d t
\end{aligned}
$$

In view of (3.23), we see that each term on the right-hand side of (3.24) tends to 0 as $n \rightarrow \infty$ uniformly for $x \in \omega$. Since the partition $\Theta$ is finite and fixed, we deduce

$$
\lim _{n \rightarrow \infty}\left[\int_{a}^{b} U_{4} d t\right]=0
$$


uniformly for $x \in \omega$, and hence also uniformly for $x \in B$, since the partition $\Omega$ is finite and fixed. Consequently, for given $a, b \in I$ and all $x \in B$ we can find $n_{2}>n_{1}$ such that for $n>n_{2}$ there holds

$$
\left|\int_{a}^{b} U_{4} d t\right| \leqslant \varepsilon
$$

Combining (3.26) with (3.21) and (3.22), we conclude that for any given $a, b \in I$ and uniformly for all $x \in B$ there holds

$$
\lim _{n \rightarrow \infty}\left(\int_{a}^{b}\left(v_{t}^{n}\left(F_{t}^{n}(x)\right)-v_{t}^{*}\left(z^{*}(t, x)\right)\right) d t\right)=0 .
$$

The equations (3.2a),(3.2b) for $F_{t}^{n}(x)$ imply

$$
F_{t}^{n}(x)=x+\int_{t_{0}}^{t}\left(v_{t}^{n}\left(F_{t}^{n}(x)\right) \quad \forall t \in I, x \in \mathbb{R}^{3} .\right.
$$

Hence, the two limits (3.18) and (3.27) show that

$$
z^{*}(t, x)=x+\int_{t_{0}}^{t}\left(v_{t}^{*}\left(z^{*}(t, x)\right)\right) \quad \forall t \in I, x \in \mathbb{R}^{3} .
$$

In view of Theorem 3.1, we see that $z^{*}$ must coincide with the unique solution $F^{*}$ of $(3.2 \mathrm{a}),(3.2 \mathrm{~b})$ determined by $v^{*}$. This proves the key convergence result (3.13).

\subsection{Necessary Optimality Conditions}

We now derive first order necessary optimality conditions in terms of the Gâteaux derivative of the objective functional $J$. Denote by $\mathrm{CR} 3=C\left(\mathbb{R}^{3}, \mathbb{R}^{3}\right)$ the vector space of continuous maps from $\mathbb{R}^{3}$ to $\mathbb{R}^{3}$ endowed with the topology of uniform convergence on bounded subsets of $\mathbb{R}^{3}$. Call MR3 the dual of CR3, i.e the space of all linear continuous maps $\Lambda:$ CR3 $\rightarrow \mathbb{R}$ which are of the form

$$
\Lambda(g)=\int_{\mathbb{R}^{3}}\langle\lambda(x), g(x)\rangle_{\mathbb{R}^{3}} d \theta(x) \quad \forall g \in \mathrm{CR} 3,
$$

where $\theta$ is any positive bounded Borel measure on $\mathbb{R}^{3}$ and $\lambda: \mathbb{R}^{3} \rightarrow \mathbb{R}^{3}$ is any Borel function with compact support such that

$$
\int_{\mathbb{R}^{3}}\|\lambda(x)\|_{\mathbb{R}^{3}} d \theta(x)<\infty .
$$

We introduce $\operatorname{Gat}(V, 3)$ as the space of all functions $v \rightarrow G^{v}$ from $L^{2}(I, V)$ into CR3 having a Gâteaux derivative $\nabla G^{v}$ at each $v \in L^{2}(I, V)$. The operators $\nabla G^{v}$ are linear maps from $L^{2}(I, V)$ to $C\left(\mathbb{R}^{3}, \mathbb{R}^{3}\right)$ of the form

$$
\nabla G^{v} \cdot w=\lim _{\varepsilon \rightarrow 0}(1 / \varepsilon)\left(G^{v+\varepsilon w}-G^{v}\right) \quad \forall w \in L^{2}(I, V) .
$$


We say that a functional $\phi:(\mathrm{CR} 3)^{q} \rightarrow \mathbb{R}, \phi$ has weak partial derivatives $\partial_{j} \phi(Z) \in \mathrm{MR} 3$ at $Z \in(\mathrm{CR} 3)^{q}$, if for any set of $q$ functions $G_{j} \in \operatorname{Gat}(V, 3)$ the composite function $f(v)=$ $\phi\left(G_{1}^{v}, \ldots, G_{q}^{v}\right)$ has a Gâteaux derivative $\nabla f(v)$ at each $v \in L^{2}(I, V)$, and the derivative in the direction $w \in L^{2}(I, V)$ is given by

$$
\nabla f(v) \cdot w=\sum_{j=1}^{q} \partial_{j} \phi(Z) \cdot\left[\nabla_{v} G_{j} \cdot w\right] \quad \forall w \in L^{2}(I, V),
$$

where $Z=\left(G_{1}^{v}, \cdots, G_{q}^{v}\right)$.

Theorem 3.3. Let $J$ be the objective functional as given by (3.9) with a disparity functional of the form $\operatorname{Disp}(v)=\phi\left(F_{t_{1}}^{v}, \ldots, F_{t_{q}}^{v}\right)$, where $\phi:\left(C\left(\mathbb{R}^{3}\right), \mathbb{R}^{3}\right)^{q} \rightarrow \mathbb{R}^{3}$ has Gâteaux partial derivatives $\partial_{j} \phi$. Assume that for all $g \in C R 3$

$$
\partial_{j} \phi . g=\int_{\mathbb{R}^{3}}<\lambda_{j}(x), g(x)>_{\mathbb{R}^{3}} d \theta_{j}(x),
$$

where $\theta_{j}, 1 \leqslant j \leqslant q$, are positive bounded Borel measures with compact support in $\mathbb{R}^{3}$ and the $\lambda_{j}: \mathbb{R}^{3} \rightarrow \mathbb{R}^{3}, 1 \leqslant j \leqslant q$, are continuous functions. Then, the Gâteaux derivative $\nabla J(v) \in L^{2}(I, V)$ is given by

$$
\nabla J(v) \cdot w=\int_{t_{0}}^{t_{q}}\left(\int_{R^{3}} w_{t} d\left(\rho_{t, v}+\eta_{t, v}\right)\right) d t
$$

where for each $t$ and $v$ the quantities $\rho_{t, v}$ and $\eta_{t, v}$ are $\mathbb{R}^{3}$-vector valued Borel measures on $\mathbb{R}^{3}$, and the measures $\eta_{t, v}$ remain constant in $t$ over each interval $\left[t_{j}, t_{j+1}\right)$. Explicit formulas for these measures are given below in the proof of this theorem.

If $v^{*} \in L^{2}(I, V)$ is a minimizing diffeomorphic flow, then $\nabla J\left(v^{*}\right)=0$, and this implies $\rho_{t, v^{*}}+\eta_{t, v^{*}}=0$ for all $t \in I$.

Proof. Obviously, the Gâteaux derivative $\nabla E(v)$ of the kinetic energy is given by

$$
\nabla E(v) . w=<v, w>_{L^{2}(I, V)} .
$$

We fix $v \in L^{2}(I, V)$ and $x \in \mathbb{R}^{3}$ and denote by $D F_{t}^{v}(x): \mathbb{R}^{3} \rightarrow \mathbb{R}^{3}$ the Jacobian $m$ of the diffeomorphism $F_{t}^{v}$ at $x$. Since

$$
\partial_{t} F_{t}^{v}(x)=v_{t}\left(F_{t}^{v}(x)\right) \text { and } \quad F_{0}^{v}(x)=x
$$

the Gâteaux derivative

$$
g_{t}=g_{t}(x, v, w)=\nabla_{v} F_{t}^{v}(x)
$$

of $F_{t}^{v}(x)$ with respect to $v$ in the direction $w \in L^{2}(I, V)$ satisfies

$$
\begin{aligned}
\partial_{t} g_{t}-D v_{t}\left(F_{t}^{v}(x)\right) \cdot g_{t} & =w_{t}\left(F_{t}^{v}(x)\right), \\
g_{0} & =0,
\end{aligned}
$$


so that $g_{t}$ is the solution of an initial-value problem for a linear ordinary differential equation with non-zero right-hand side $r_{t}=r_{t}(x, v, w)=w_{t}\left(F_{t}^{v}(x)\right)$. Setting $p_{t}=p_{t}(x, v)=$ $D v_{t}\left(F_{t}^{v}(x)\right)$, this initial-value problem can be written as

$$
\begin{aligned}
\partial_{t} g_{t} & =p_{t} \cdot g_{t}+m_{t}, \\
g_{0} & =0 .
\end{aligned}
$$

We denote by $R_{s, t}, t_{0}<s<t<t_{q}$ the resolvent of the homogeneous linear ordinary differential equation $\partial_{t} z_{t}=p_{t} . z_{t}$ which satisfies

$$
\partial_{t} R_{s, t}=p_{t} \cdot R_{s, t} \quad \text { and } \quad R_{s, s}=\mathrm{Id} \quad \forall s<t .
$$

We note that $R_{s, t}$ depends only on $s, t, x, v$ and that the solution $g_{t}$ of $(3.35 \mathrm{a}),(3.35 \mathrm{~b})$ is then given by

$$
g_{t}=\int_{t_{0}}^{t} R_{s, t} \cdot m_{s} d s
$$

With a slight change of notations, omitting the explicit dependence on $x \in \mathbb{R}^{3}$, this results in

$$
\nabla_{v} F_{t}^{v} \cdot w=\int_{t_{0}}^{t} R_{s, t}^{v} \cdot w_{s}\left(F_{s}^{v}\right) d s .
$$

In view of the hypothesis on the functional $\phi$, it follows that

$$
\left(\nabla \operatorname{Disp}^{v}\right) \cdot w=\sum_{j=1}^{q} \partial_{j} \phi \cdot \nabla_{v} F_{t_{j}}^{v} \cdot w
$$

and hence, taking (3.36) into account, we have

$$
\left[\nabla \operatorname{Disp}^{v}\right] \cdot w=\sum_{j=1}^{q} \int_{t_{0}}^{t_{j}}\left[\partial_{j} \Phi \cdot R_{s, t_{j}}^{v}\right] \cdot\left[w_{s}\left(F_{s}^{v}\right)\right] d s .
$$

For each $s \in I$ and fixed $v \in L^{2}(I, V)$ we define the linear map $A_{s}: V \rightarrow \mathbb{R}$ by

$$
A_{s}^{v}=\sum_{j=1}^{q} 1_{\left[t_{0}, t_{j}\right]}(s) \partial_{j} \phi R_{s, t_{j}}^{v}
$$

whence

$$
[\nabla \operatorname{Disp}(v)] \cdot w=\int_{t_{0}}^{t_{q}} A_{s}^{v} \cdot\left[w_{s}\left(F_{s}^{v}\right)\right] d s .
$$

Finally, due to (3.34) we obtain

$$
\nabla J(v) \cdot w=\int_{t_{0}}^{t_{q}}\left[<v_{t}, w_{t}>_{V}+A_{t}^{v} \cdot\left[w_{t}\left(F_{t}^{v}\right)\right]\right] d t .
$$


We fix an instant $s<t_{j}$ and for $x \in \mathbb{R}^{3}$ temporarily define the $3 \times 3$ matrix $R(x)$ and the diffeomorphism $x \rightarrow u(x) \in \mathbb{R}^{3}$ according to

$$
R(x)=R_{s, t_{j}}^{v}(x) \quad \text { and } \quad u(x)=F_{s}^{v}(x) .
$$

For the function $g: x \rightarrow g(x)=R(x) w_{s}(u(x))$ we thus get

$$
\partial_{j} \phi \cdot\left[R_{s, t_{j}}^{v} \cdot\left[w_{s}\left(F_{s}^{v}\right)\right]=\int_{R^{3}}<\lambda_{j}(x), R(x) w_{s}(u(x))>_{\mathbb{R}^{3}} d \theta_{j}(x) .\right.
$$

We define the bounded measure $\mu_{j}=u\left(\theta_{j}\right)$ as the direct image of the measure $\theta_{j}$ by the diffeomorphism $u$ and note that $\mu_{j}$ depends only on $j, s, v$, which are fixed temporarily. In view of the definition of transported measures, we have

$$
\int_{\mathbb{R}^{3}}\left\langle\lambda_{j}(x), R(x) w_{s}(u(x))\right\rangle_{\mathbb{R}^{3}} d \theta_{j}(x)=\int_{\mathbb{R}^{3}}\left\langle R^{T}\left(u^{-1}(y)\right) \lambda_{j}\left(u^{-1}(y)\right), w_{s}(y)\right\rangle_{\mathbb{R}^{3}} d \mu_{j}(y),
$$

where $R^{T}$ stands for the transpose of the matrix $R$.

Now, for $s<t_{j}$ we define

$$
\begin{aligned}
a_{j, s, v}(x) & :=\left[R_{s, t_{j}}^{v}(x)\right]^{T} \cdot \lambda_{j}(x) \quad \forall x \in \mathbb{R}^{3}, \\
b_{j, s, v}(y) & :=a_{j, s, v} \circ\left[F_{s}^{v}\right]^{-1}, \\
\mu_{j, s, v} & :=F_{s}^{v}\left[\theta_{j}\right] .
\end{aligned}
$$

It follows that

$$
A_{s}^{v} \cdot\left[w_{s}\left(F_{s}^{v}\right)\right]=\sum_{j=1}^{q} 1_{\left[t_{0}, t_{j}\right]}(s) \int_{\mathbb{R}^{3}}\left\langle b_{j, s, v}(y), w_{s}(y)\right\rangle_{\mathbb{R}^{3}} d \mu_{j, s, v}(y) .
$$

We introduce the vector-valued Borel measure $\nu_{j, s, v}$, taking values in $\mathbb{R}^{3}$, as the measure with vector-valued density $b_{j, s, v}$ with respect to the bounded Borel measure $\mu_{j, s, v}$, so that for any continuous function $g: \mathbb{R}^{3} \rightarrow \mathbb{R}^{3}$ there holds

$$
\left.\int_{\mathbb{R}^{3}} g d \nu_{j, s, v}=\int_{\mathbb{R}^{3}}\left\langle b_{j, s, v}(y)\right), w_{s}(y)\right\rangle_{\mathbb{R}^{3}} d \mu_{j, s, v} .
$$

We then define the vector-valued measure $\eta_{s, v}$ on $\mathbb{R}^{3}$ by means of

$$
\eta_{s, v}=\sum_{j=1}^{q} 1_{\left[t_{0}, t_{j}\right]}(s) \nu_{j, s, v}
$$

and thus obtain

$$
\nabla J(v) \cdot w=\int_{t_{0}}^{t_{q}}\left(\left\langle v_{t}, w_{t}\right\rangle_{V}+\int_{\mathbb{R}^{3}} w_{t} d \eta_{t, v}\right) d t .
$$

On the Hilbert space $V$, the norm and the scalar product are defined by the kernel $K(x, y)$, whence

$$
\left\langle v_{t}, w_{t}\right\rangle_{V}=\int_{\mathbb{R}^{3}}\left\langle K v_{t}(x), w_{t}(x)\right\rangle_{\mathbb{R}^{3}} d x=\int_{\mathbb{R}^{3}} w_{t} d \rho_{t, v} .
$$


Here, the vector-valued measure $\rho_{t, v}$ has density $K v_{t}$ with respect to the Lebesgue measure on $\mathbb{R}^{3}$. Finally, we obtain the following representation which is valid for all $w \in L^{2}(I, V)$

$$
\nabla J(v) \cdot w=\int_{t_{0}}^{t_{q}}\left(\int_{\mathbb{R}^{3}} w_{t} d\left(\rho_{t, v}+\eta_{t, v}\right)\right) d t
$$

Let $v^{*} \in L^{2}(I, V)$ be a minimizer of the objective functional $J$. Obviously, we must have

$$
\nabla J\left(v^{*}\right) . w=0 \quad \forall w \in L^{2}(I, V) .
$$

In view of (3.42), this forces the measures $\rho_{t, v}+\eta_{t, v}$ to be zero for all $t \in I$ except for a possible exceptional set $\Omega \subset I$ of Lebesgue measure zero. Since the measures $\eta_{t, v}$ are constant in $t$ within each interval $\left[t_{j}, t_{j+1}\right)$ and the measures $\rho_{t, v}$ are continuous in $t$, we conclude that $\Omega$ must be empty.

\section{Dirac Measures and Diffeomorphic Point Matching}

Diffeomorphic point matching $[13,24,27]$ is a particular case of diffeomorphic matching of measures that can be derived from the general framework of section 3. In such a framework, a given sequence of $q+1$ instantaneous shape snapshots $S_{j}=S_{t_{j}}$ at fixed time frames $t_{j}$, $j=0, \cdots, q$, is identified by a family of point sets $X_{j}=\left\{x_{1}^{j}, \ldots, x_{N_{j}}^{j}\right\}$. Let $\hat{S}_{j}=F_{t_{j}}^{v}\left(S_{0}\right)$ be a sequence of $q$ submanifolds generated at instants $t_{j}, 1 \leqslant j \leqslant q$, from the initial snapshot $S_{0}$ by a $\mathbb{R}^{3}$-diffeomorphism $F_{t}^{v}$ satisfying (3.2a),(3.2b) with unknown flow dynamics $v \in L^{2}(I, V)$. Let $\hat{X}_{j}=F_{t_{j}}^{v}\left(X_{0}\right)=\left\{F_{t_{j}}^{v}\left(x_{1}^{0}\right), \ldots, F_{t_{j}}^{v}\left(x_{N_{0}}^{0}\right)\right\}$ be the sequence of $q$ point sets generated by $F_{t}^{v}$ at instants $t_{j}, 1 \leqslant j \leqslant q$ from the initial point set $X_{0}$. We denote by $x_{n}(t)=F_{t}^{v}\left(x_{n}^{0}\right)$, $t \in I$, the corresponding $N_{0}$ trajectories emanating from $x_{n}^{0}, 1 \leqslant n \leqslant N_{0}$, at $t=0$. Thus we have $\hat{X}_{j}=\left\{x_{1}\left(t_{j}\right), \ldots x_{N_{0}}\left(t_{j}\right)\right\}, 1 \leqslant j \leqslant q$. It is natural to represent $S_{j}$ and $\hat{S}_{j}, 1 \leqslant j \leqslant q$, as weighted sums of Dirac measures $\delta_{x_{m}^{j}}, 1 \leqslant m \leqslant N_{j}$, and $\delta_{x_{n}\left(t_{j}\right)}, 1 \leqslant n \leqslant N_{0}$, associated with the point sets $X_{j}$ and $\hat{X}_{j}$. In particular, we assume

$$
\mu_{S_{j}}=\sum_{m=1}^{N_{j}} b_{m}^{j} \delta_{x_{m}^{j}}, \quad \mu_{\hat{S}_{j}}=\sum_{n=1}^{N_{0}} a_{n} \delta_{x_{n}\left(t_{j}\right)}, \quad a_{n}, b_{m}^{j} \in \mathbb{R} \quad, \quad j=1, \cdots, q .
$$

It follows that the disparity cost functional (3.7) takes the form

$$
\operatorname{Disp}(v)=\sum_{j=1}^{q} \lambda_{j} D_{K_{\sigma_{j}}}^{2}\left(\hat{S}_{j}, S_{j}\right)=\sum_{j=1}^{q} \lambda_{j}\left\|\mu_{\hat{S}_{j}}-\mu_{S_{j}}\right\|_{K_{\sigma_{j}}}^{2} .
$$

The terms $\left\|\mu_{\hat{S}_{j}}-\mu_{S_{j}}\right\|_{K_{\sigma_{j}}}^{2}, 1 \leqslant j \leqslant q$, represent the Borel distances between the shapes $S_{j}$ and $\hat{S}_{j}$ associated with radial Gaussian kernels $K_{\sigma_{j}}$ for suitable scale parameters $\sigma_{j}>0$. From (3.5) and (3.6), we have

$$
\left\|\mu_{\hat{S}_{j}}-\mu_{S_{j}}\right\|_{K_{\sigma_{j}}}^{2}=\left\langle\mu_{\hat{S}_{j}}-\mu_{S_{j}}, \mu_{\hat{S}_{j}}-\mu_{S_{j}}\right\rangle_{K_{\sigma_{j}}}=\left\langle\mu_{\hat{S}_{j}}, \mu_{\hat{S}_{j}}\right\rangle_{K_{\sigma_{j}}}-2\left\langle\mu_{\hat{S}_{j}}, \mu_{S_{j}}\right\rangle_{K_{\sigma_{j}}}+\left\langle\mu_{S_{j}}, \mu_{S_{j}}\right\rangle_{K_{\sigma_{j}}},
$$


where

$$
\begin{aligned}
\left\langle\mu_{\hat{S}_{j}}, \mu_{\hat{S}_{j}}\right\rangle_{K_{\sigma_{j}}} & =\int_{\mathbb{R}^{3}} \int_{\mathbb{R}^{3}} K_{\sigma_{j}}\left(x, x^{\prime}\right) d \mu_{\hat{S}_{j}}(x) d \mu_{\hat{S}_{j}}\left(x^{\prime}\right)=\sum_{n=1}^{N_{0}} \sum_{n^{\prime}=1}^{N_{0}} a_{n} a_{n^{\prime}} K_{\sigma_{j}}\left(x_{n}\left(t_{j}\right), x_{n^{\prime}}\left(t_{j}\right)\right), \\
\left\langle\mu_{\hat{S}_{j}}, \mu_{S_{j}}\right\rangle_{K_{\sigma_{j}}} & =\int_{\mathbb{R}^{3}} \int_{\mathbb{R}^{3}} K_{\sigma_{j}}\left(x, x^{\prime}\right) d \mu_{\hat{S}_{j}}(x) d \mu_{S_{j}}\left(x^{\prime}\right)=\sum_{n=1}^{N_{0}} \sum_{m=1}^{N_{j}} a_{n} b_{m}^{j} K_{\sigma_{j}}\left(x_{n}\left(t_{j}\right), x_{m}^{j}\right), \\
\left\langle\mu_{S_{j}}, \mu_{S_{j}}\right\rangle_{K_{\sigma_{j}}} & =\int_{\mathbb{R}^{3}} \int_{\mathbb{R}^{3}} K_{\sigma_{j}}\left(x, x^{\prime}\right) d \mu_{S_{j}}(x) d \mu_{S_{j}}\left(x^{\prime}\right)=\sum_{m=1}^{N_{j}} \sum_{m^{\prime}=1}^{N_{j}} b_{m}^{j} b_{m^{\prime}}^{j} K_{\sigma_{j}}\left(x_{m}^{j}, x_{m^{\prime}}^{j}\right) .
\end{aligned}
$$

Recall that $x_{n}(t)=F_{t}^{v}\left(x_{n}^{0}\right), t \in I, 1 \leqslant n \leqslant N_{0}$, are the solutions of the ODEs

$$
\begin{aligned}
\frac{d x_{n}(t)}{d t} & =v_{t}\left(x_{n}(t)\right), \quad t \in(0,1], \\
x_{n}(0) & =x_{n}^{0} .
\end{aligned}
$$

Thus the trajectories $x_{n}(t), 1 \leqslant n \leqslant N_{0}$, and the disparity cost functional $\operatorname{Disp}(v)$, are uniquely determined by the values of $v_{t}$ taken at $N_{0}$ points $x_{n}(t)$. Taking into account that $V=V_{K}$ is a RKHS associated to a radial Gaussian kernel $K=K_{\sigma_{0}}$, this allows us to restrict the search for $v_{t} \in V$ to the set of linear combination of $K_{x_{n}(t)}$, $1 \leqslant n \leqslant N_{0}$, and thus places us in a finite dimensional situation. We look for the flow $v_{t}$ under the form $v_{t}=\sum_{n=1}^{N_{0}} \alpha_{n}(t) K_{x_{n}(t)}, \alpha_{n}(t) \in \mathbb{R}^{3}$, which may also be written

$$
v_{t}(x)=\sum_{n=1}^{N_{0}} K_{\sigma_{0}}\left(x_{n}(t), x\right) \alpha_{n}(t) \quad, \quad \forall x \in \mathbb{R}^{3} .
$$

By the self reproducing property of $K$, we have

$$
\left\|v_{t}\right\|_{V}^{2}=\sum_{n=1}^{N_{0}} \sum_{n^{\prime}=1}^{N_{0}} K_{\sigma_{0}}\left(x_{n}(t), x_{n^{\prime}}(t)\right) \alpha_{n}^{T}(t) \alpha_{n^{\prime}}(t) .
$$

We introduce the matrix-vector notations:

$$
\begin{aligned}
& x^{(0)}=\left(x_{1}^{(0)}, \cdots, x_{N_{0}}^{(0)}\right)^{T} \in \mathbb{R}^{N_{0} d}, \quad x(t)=\left(x_{1}(t), \cdots, x_{N_{0}}(t)\right)^{T} \in \mathbb{R}^{N_{0} d}, \quad t \in I, \\
& \alpha(t):=\left(\alpha_{1}(t), \cdots, \alpha_{N_{0}}(t)\right)^{T} \in \mathbb{R}^{N_{0} d}, \quad t \in I, \\
& A(x(t))=\left(A_{n n^{\prime}}(x(t))\right)_{n, n^{\prime}=1}^{N_{0}} \in \mathbb{R}^{N_{0} d \times N_{0} d}, A_{n n^{\prime}}(x(t)):=K_{\sigma_{0}}\left(x_{n}(t), x_{n^{\prime}}(t)\right) I_{d} \in \mathbb{R}^{d \times d} .
\end{aligned}
$$

It follows that the kinetic energy $E(v)$ defined by (3.1) takes the form

$$
E(v)=\frac{1}{2} \int_{0}^{1} \alpha(t)^{T} A(x(t)) \alpha(t) d t
$$

Hence, in terms of $\alpha \in L^{2}\left(I, \mathbb{R}^{N_{0} d}\right)$, the objective functional reads

$$
J(\alpha)=\frac{1}{2} \int_{0}^{1} \alpha(t)^{T} A(x(t)) \alpha(t) d t+\sum_{j=1}^{q} \lambda_{j} \operatorname{Disp}_{j}\left(x\left(t_{j}\right)\right)
$$


where the $q$ disparity functions $\operatorname{Disp}_{j}\left(x\left(t_{j}\right)\right)=\left\|\mu_{\hat{S}_{j}}-\mu_{S_{j}}\right\|_{K_{\sigma_{j}}}^{2}, 1 \leqslant j \leqslant q$ are given by the right-hand sides in (4.2) and (4.3). The diffeomorphic point matching amounts to the solution of the optimal control problem

$$
\inf _{\alpha \in L^{2}\left(I, \mathbb{R}^{N_{0}}\right)} J(\alpha)
$$

subject to

$$
\begin{aligned}
\frac{d x(t)}{d t} & =A(x(t)) \alpha(t), \quad t \in(0,1] \\
x(0) & =x^{(0)}
\end{aligned}
$$

The existence of a solution $\alpha^{*}$ of (4.8a)-(4.8c) follows from Theorem 3.2, whereas the first order necessary optimality conditions can be either derived from Theorem 3.3 by evaluating the terms in (3.33) within the current setting (cf., e.g., [21]) or directly as will be shown in the proof of the following result.

Theorem 4.1. Assume that $\alpha^{*}(\cdot)$ is the solution of the optimal control problem (4.8), and that $x^{*}(\cdot)$ is the corresponding trajectory. Then there exists a function $p^{*}(\cdot)$, called the adjoint state, such that the triple $\left(x^{*}, p^{*}, \alpha^{*}\right)$ satisfies

$$
\begin{gathered}
\frac{d x^{*}(t)}{d t}=A\left(x^{*}(t)\right) \alpha^{*}(t), \quad t \in(0,1], \\
x^{*}(0)=x^{(0)}, \\
-\frac{d p^{*}(t)}{d t}=B\left(x^{*}(t), \alpha^{*}(t)\right)^{T}\left(p^{*}(t)+\frac{1}{2} \alpha^{*}(t)\right), \quad t \in\left(t_{j-1}, t_{j}\right), \\
p^{*}\left(t_{q}^{+}\right)=0, p^{*}\left(t_{j}^{-}\right)=p^{*}\left(t_{j}^{+}\right)+\lambda_{j} \nabla \operatorname{Disp}_{j}\left(x^{*}\left(t_{j}\right)\right) \quad, \quad j=q, \cdots, 1, \\
A\left(x^{*}(t)\right)\left(\alpha^{*}(t)+p^{*}(t)\right)=0, \quad t \in(0,1] .
\end{gathered}
$$

In the adjoint state equation (4.10a), the matrix

$$
B\left(x^{*}(t), \alpha^{*}(t)\right)=\nabla_{x}\left(A\left(x^{*}(t)\right) \alpha^{*}(t)\right),
$$

is given by

$$
\begin{aligned}
& \left.B\left(x^{*}(t), \alpha^{*}(t)\right)=B_{n m}\left(x^{*}(t), \alpha^{*}(t)\right)\right)_{n, m=1}^{N_{0}} \in \mathbb{R}^{N_{0} d \times N_{0} d}, \\
& B_{n m}\left(x^{*}(t), \alpha^{*}(t)\right):=\alpha_{m}^{*}(t)\left(\nabla_{2} K_{\sigma_{0}}\left(x_{n}^{*}(t), x_{m}^{*}(t)\right)\right)^{T}+\delta_{n m} \sum_{k=1}^{N} \alpha_{k}^{*}(t)\left(\nabla_{1} K_{\sigma_{0}}\left(x_{n}^{*}(t), x_{k}^{*}(t)\right)\right)^{T},
\end{aligned}
$$

where $\nabla_{x}$ denotes the gradient with respect the argument $x(t)$ and $\nabla_{i} K_{\sigma_{0}}(\cdot, \cdot), 1 \leqslant i \leqslant 2$, stands for the gradient with respect to the $i$-th argument of $K_{\sigma_{0}}(\cdot, \cdot)$.

Moreover, (4.10b) represents the jump discontinuities of $p^{*}(\cdot)$ at times $t_{j}, 1 \leqslant j \leqslant q$. 
Proof. Introducing Lagrange multipliers $p(t)=\left(p_{1}(t), \cdots, p_{N_{0}}(t)\right)^{T} \in \mathbb{R}^{N_{0} d}, t \in I$, the Lagrangian associated with (4.8a)-(4.8c) is given by

$$
\begin{aligned}
& L(\alpha, x, p):=J(\alpha)-\int_{0}^{1} p \cdot\left(\frac{d x}{d t}-A(x(t)) \alpha(t)\right) d t \\
& =-\int_{0}^{1} p \cdot \frac{d x}{d t} d t+\int_{0}^{1}(p+\alpha / 2) \cdot A(t, x) \alpha d t+\sum_{j=1}^{q} \lambda_{j} \operatorname{Disp}_{j}\left(x\left(t_{j}\right)\right) .
\end{aligned}
$$

The optimality conditions for a critical point $\left(\alpha^{*}, x^{*}, p^{*}\right)$ of $L(\alpha, x, p)$ read as follows:

$$
\begin{aligned}
& L_{\alpha}\left(\alpha^{*}, x^{*}, p^{*}\right)=0, \\
& L_{x}\left(\alpha^{*}, x^{*}, p^{*}\right)=0, \\
& L_{p}\left(\alpha^{*}, x^{*}, p^{*}\right)=0 .
\end{aligned}
$$

Obviously, (4.12a) implies (4.11), whereas (4.12c) gives rise to (4.9). Using integration by parts

$$
\begin{aligned}
-\int_{0}^{1} p \cdot \frac{d x}{d t} d t & =-\sum_{j=1}^{q} \int_{t_{j-1}}^{t_{j}} p \cdot \frac{d x}{d t} d t=\sum_{j=1}^{q}\left(\int_{t_{j-1}}^{t_{j}} \frac{d p}{d t} \cdot x d t-p\left(t_{j}^{-}\right) \cdot x\left(t_{j}\right)+p\left(t_{j-1}^{+}\right) \cdot x\left(t_{j-1}\right)\right) \\
& =\int_{0}^{1} \frac{d p}{d t} \cdot x d t+\sum_{j=1}^{q-1}\left(-p\left(t_{j}^{-}\right)+p\left(t_{j}^{+}\right)\right) \cdot x\left(t_{j}\right)-p(1) \cdot x(1)+p(0) \cdot x(0),
\end{aligned}
$$

(4.12a) yields (4.10).

\section{Numerical Solutions for Diffeomorphic Matching of Multiple Snapshots}

In this section, we outline the basic matching algorithm based on gradient descent and time discretizations of the optimality conditions (4.9a),(4.9b) and (4.10a),(4.10b) as well as variants involving iteration-dependent weighting parameters for the matching term in the objective functional. We will also summarize several initialization schemes.

\subsection{The Basic Matching Algorithm}

For the time discretizations of the optimal control problem (4.8) we introduce a partition $\Delta_{I}$ of $I$ according to

$$
\Delta_{I}:=\cup_{j=1}^{q} \Delta_{I_{j}}, \quad \Delta_{I_{j}}:=\left\{t_{j-1}=: t^{L_{j-1}}<t^{L_{j-1}+1}<\cdots<t^{L_{j}-1}<t^{L_{j}}:=t_{j}\right\},
$$

where $\Delta_{I_{j}}, 1 \leqslant j \leqslant q$, are subpartitions of intervals $I_{j}=\left[t_{j-1}, t_{j}\right]$ whose endpoints $t_{j}$ are key time frames at which the shape snapshots $S_{j}=S\left(t_{j}\right)$ are given. We set $L_{0}:=0$ and $L:=L_{q}$ and define step sizes $\Delta t^{\ell}:=t_{\ell+1}-t_{\ell}>0,0 \leqslant \ell \leqslant L-1$.

We introduce the discrete control space

$$
\mathcal{U}^{\Delta_{I}}=\mathbb{R}^{L \times\left(N_{0} d\right)}
$$


equipped with the inner product

$$
(\boldsymbol{\alpha}, \boldsymbol{\beta})_{\Delta_{I}}=\sum_{\ell=0}^{L-1} \Delta t^{\ell} \boldsymbol{\alpha}^{\ell} \cdot \boldsymbol{\beta}^{\ell}=\sum_{\ell=0}^{L-1} \sum_{n=1}^{N_{0}} \Delta t^{\ell} \alpha_{n}^{\ell} \cdot \beta_{n}^{\ell},
$$

and discretize the state equation (4.9a),(4.9b) and the adjoint state equation (4.10a),(4.10b) by the explicit Euler method. Introducing the notations

$$
\begin{aligned}
& \mathbf{x}=\left\{\mathbf{x}^{\ell}\right\}_{\ell=0}^{L}, \quad \mathbf{x}^{\ell}=\left\{x_{n}^{\ell}\right\}_{n=1}^{N_{0}}, \quad x_{n}^{\ell} \approx x_{n}\left(t^{\ell}\right), \\
& \mathbf{p}=\left\{\mathbf{p}^{\ell}\right\}_{\ell=0}^{L-1}, \quad \mathbf{p}^{\ell}=\left\{p_{n}^{\ell}\right\}_{n=1}^{N_{0}}, \quad p_{n}^{\ell} \approx p_{n}\left(t^{\ell}\right), \\
& \boldsymbol{\alpha}=\left\{\boldsymbol{\alpha}^{\ell}\right\}_{\ell=0}^{L-1}, \quad \boldsymbol{\alpha}^{\ell}=\left\{\alpha_{n}^{\ell}\right\}_{n=1}^{N_{0}}, \quad \alpha_{n}^{\ell} \approx \alpha_{n}\left(t^{\ell}\right),
\end{aligned}
$$

the discretized optimality system reads

$$
\begin{gathered}
\frac{\mathbf{x}^{\ell+1}-\mathbf{x}^{\ell}}{\Delta t^{\ell}}=A\left(\mathbf{x}^{\ell}\right) \boldsymbol{\alpha}^{\ell}, \quad \ell=0, \cdots, L-1, \\
\mathbf{x}^{0}=\mathbf{x}^{(0)}, \\
\frac{\mathbf{p}^{\ell-1}-\mathbf{p}^{\ell}}{\Delta t^{\ell}}=B\left(\mathbf{x}^{\ell}, \boldsymbol{\alpha}^{\ell}\right)^{T}\left(\mathbf{p}^{\ell}+\frac{1}{2} \boldsymbol{\alpha}^{\ell}\right), \quad \ell=L_{j}-1, \cdots, L_{j-1}, \\
\mathbf{p}^{L_{q}-1}=0, \mathbf{p}^{L_{j}-1}=\mathbf{p}^{L_{j}-1}+\lambda_{j} \nabla \operatorname{Disp}_{j}\left(\mathbf{x}^{L_{j}}\right), \quad j=q, \cdots, 1 \\
A\left(\mathbf{x}^{\ell}\right)\left(\boldsymbol{\alpha}^{\ell}+\mathbf{p}^{\ell}\right)=\mathbf{0} \quad, \quad 0 \leqslant \ell \leqslant L-1 .
\end{gathered}
$$

The condition (5.5b), representing the jump discontinuities of $\mathbf{p}^{L_{j}-1}$ at discrete times $t^{L_{j}-1}$ $\left(=t^{L_{j}}-\Delta t^{L_{j}}\right)$, is the discrete version of (4.10b) for jump discontinuities of $p^{*}(\cdot)$ at snapshot time frames $t_{j}\left(=t^{L_{j}}\right), 1 \leqslant j \leqslant q$. This time backward shift of jump discontinuities by steplength $\Delta t^{L_{j}}$ stems from our choice of the explicit Euler method for the time discretization of the state equation (5.4a) and the adjoint state equation (5.5a). It turns out that (5.4)-(5.6) represent the optimality conditions for a discrete minimization problem. In fact, introducing $J^{\Delta_{I}}(\boldsymbol{\alpha})$ as the discrete objective functional

$$
J^{\Delta_{I}}(\boldsymbol{\alpha}):=\sum_{l=0}^{L-1} \frac{\Delta t^{\ell}}{2}\left(\boldsymbol{\alpha}^{\ell}\right)^{T} A\left(\mathbf{x}^{\ell}\right) \boldsymbol{\alpha}^{\ell}+\sum_{j=1}^{q} \lambda_{j} \operatorname{Disp}_{j}\left(\mathbf{x}^{L_{j}}\right),
$$

we have the following result.

Theorem 5.1. The equations (5.4)-(5.6) are the first order necessary optimality conditions for the finite dimensional minimization problem

$$
\min _{\boldsymbol{\alpha} \in \mathcal{U}^{\Delta_{I}}} J^{\Delta_{I}}(\boldsymbol{\alpha})
$$

subject to the discrete state equations (5.4a),(5.4b).

Proof. The proof is the discrete analogue of the proof of Theorem 4.1 and will thus be omitted. 
Corollary 5.2. Let $\left(\mathbf{x}^{*}, \mathbf{p}^{*}, \boldsymbol{\alpha}^{*}\right)$ with $\mathbf{x}^{*}=\left\{\mathbf{x}_{*}^{\ell}\right\}_{\ell=0}^{L}, \mathbf{p}^{*}=\left\{\mathbf{p}_{*}^{\ell}\right\}_{\ell=0}^{L}, \boldsymbol{\alpha}^{*}=\left\{\boldsymbol{\alpha}_{*}^{\ell}\right\}_{\ell=0}^{L}$ satisfy the discrete optimality system (5.4)-(5.6). Then, it holds

$$
\mathbf{0}=\boldsymbol{\nabla} J^{\Delta_{I}}\left(\boldsymbol{\alpha}^{*}\right)
$$

where

$$
\nabla J^{\Delta_{I}}\left(\boldsymbol{\alpha}^{*}\right)=\left\{\mathbf{g}^{\ell}\right\}_{\ell=0}^{L-1}, \quad \mathbf{g}^{\ell}=A\left(\mathbf{x}^{\ell}\right)\left(\boldsymbol{\alpha}_{*}^{\ell}+\mathbf{p}_{*}^{\ell}\right) .
$$

Proof. We observe that

$$
\delta J^{\Delta_{I}}(\boldsymbol{\alpha})=\left(\nabla J^{\Delta_{I}}(\boldsymbol{\alpha}), \delta \boldsymbol{\alpha}\right)_{\Delta I}
$$

From (5.7) we deduce

$$
\begin{aligned}
\delta J^{\Delta_{I}}(\boldsymbol{\alpha}) & =\sum_{l=0}^{L-1} \Delta t^{\ell}\left(\left(\boldsymbol{\alpha}^{\ell}\right)^{T} A\left(\mathbf{x}^{\ell}\right) \delta \boldsymbol{\alpha}^{\ell}+\frac{1}{2}\left(\boldsymbol{\alpha}^{\ell}\right)^{T} B\left(\mathbf{x}^{\ell}, \boldsymbol{\alpha}^{\ell}\right) \delta \mathbf{x}^{\ell}\right) \\
& +\sum_{j=1}^{q} \lambda_{j} \nabla \operatorname{Disp}_{j}\left(\mathbf{x}^{L_{j}}\right) \delta \mathbf{x}^{L_{j}}
\end{aligned}
$$

where

$$
\begin{aligned}
& \frac{\delta \mathbf{x}^{\ell+1}-\delta \mathbf{x}^{\ell}}{\Delta t^{\ell}}=A\left(\mathbf{x}^{\ell}\right) \delta \boldsymbol{\alpha}^{\ell}+B\left(\mathbf{x}^{\ell}, \boldsymbol{\alpha}^{\ell}\right) \delta \mathbf{x}^{\ell}, \quad \ell=0, \cdots, L-1, \\
& \delta \mathbf{x}^{0}=\mathbf{0}
\end{aligned}
$$

Multiplying both sides of $(5.12 \mathrm{~b})$ by $\mathbf{p}^{\ell}$, partial summation yields

$$
\begin{aligned}
0= & \sum_{l=0}^{L-1} \Delta t^{\ell} \mathbf{p}^{\ell} \cdot\left(\frac{\delta \mathbf{x}^{\ell+1}-\delta \mathbf{x}^{\ell}}{\Delta t^{\ell}}-A\left(\mathbf{x}^{\ell}\right) \delta \boldsymbol{\alpha}^{\ell}-B\left(\mathbf{x}^{\ell}, \boldsymbol{\alpha}^{\ell}\right) \delta \mathbf{x}^{\ell}\right) \\
= & \sum_{l=1}^{L-1} \Delta t^{\ell} \frac{\mathbf{p}^{\ell-1}-\delta \mathbf{p}^{\ell}}{\Delta t^{\ell}} \cdot \delta \mathbf{x}^{\ell}+\mathbf{p}^{L-1} \cdot \delta \mathbf{x}^{L}-\mathbf{p}^{0} \cdot \delta \mathbf{x}^{0}-\sum_{l=0}^{L-1} \Delta t^{\ell} \mathbf{p}^{\ell} \cdot A\left(\mathbf{x}^{\ell}\right) \delta \boldsymbol{\alpha}^{\ell} \\
& -\sum_{l=0}^{L-1} \Delta t^{\ell} \mathbf{p}^{\ell} \cdot B\left(\mathbf{x}^{\ell}, \boldsymbol{\alpha}^{\ell}\right) \delta \mathbf{x}^{\ell} .
\end{aligned}
$$

If we take (5.5a),(5.5b) into account, it follows from (5.13), (5.12a) that

$$
\delta J^{\Delta_{I}}(\boldsymbol{\alpha})=\sum_{l=0}^{L-1} \Delta t^{\ell} A\left(\mathbf{x}^{\ell}\right)\left(\boldsymbol{\alpha}^{\ell}+\mathbf{p}^{\ell}\right) \cdot \delta \boldsymbol{\alpha}^{\ell} .
$$

Since $\delta \boldsymbol{\alpha}=\left\{\delta \boldsymbol{\alpha}^{\ell}\right\}_{\ell=0}^{L-1}$ is arbitrary, (5.11) results in

$$
\nabla J^{\Delta_{I}}(\boldsymbol{\alpha})=\left\{\mathbf{g}^{\ell}\right\}_{\ell=0}^{L-1}, \quad \mathbf{g}^{\ell}=A\left(\mathbf{x}^{\ell}\right)\left(\boldsymbol{\alpha}^{\ell}+\mathbf{p}^{\ell}\right) .
$$

In view of of (5.9),(5.10), the discrete minimization problem (5.8) can be solved by a gradient based algorithm operating in $\mathcal{U}^{\Delta_{I}}$. 


\subsection{Diffeomorphic Matching for Multiple Snapshots of 3D Curves and Surfaces}

We compute a diffeomorphic matching for multiple snapshots of 3D curves, 3D surfaces, or finite unions of 3D-curves and surfaces, by solving the minimization problem (5.8) with $q$ weighting factors $\lambda_{j}=\lambda, 1 \leqslant j \leqslant q$, in the objective functional (5.7), where the weights help balance the various matching accuracies desirable for the $q$ given snapshots.

The gradient descent algorithms we have implemented and tested all use an outer/inner iterative scheme which features a continuation method in the regularization parameter $\lambda$ as outer iterations and a gradient method with Armijo line search (cf., e.g., [2]) as inner iterations.

The continuation method plays an essential and efficient part : the regularization parameter $\lambda$ is initialized at a low value and is increased by moderate multiplicative steps until the $q$ geometric matching disparities with the given $q$ snapshots have all reached a preassigned low target level. After each multiplicative increase of $\lambda$, the gradient $G$ of the objective function jumps up in norm, and we keep $\lambda$ fixed during an "inner" iteration of the gradient descent, until the norm of $G$ reaches again a low preassigned value. The value of $\lambda$ is then increased again ("outer" iteration).

At the end of the inner gradient descent iteration performed at a fixed value $\lambda$, the kinetic energy and the disparity term in the objective function $J=K i n+\lambda D i s p$ reach terminal values $K i n_{\lambda}$ and $D i s p_{\lambda}$. In $R^{2}$, the points $\left[K i n_{\lambda}, D i s p_{\lambda}\right]$ define a curve $\Gamma \subset R^{2}$ parametrized by $\lambda>0$ called the Pareto frontier of the objective function $J$. The convexity of the Pareto frontier is usually a desirable feature for regularized optimization problems, and we have empirically observed this convexity in all the multiple snapshots matching applications we have studied below.

In the applications presented below, the given snapshots $S_{j}=S_{t_{j}} \subset R^{3}, 0 \leqslant j \leqslant q$, are assumed to have been generated at fixed instants $t=t_{j}$ by unknown diffeomorphic deformations $S(t)$ of a known initial deformable shape $S(0)$. The initial shape $S(0)$ is a finite union of geometric components $C_{i}$, where each $C_{i}$ is either a segment of piecewise smooth 3D-curve, or a piecewise smooth 3D-surface with piecewise smooth boundary. Pairs $C_{i}, C_{k}$ of components may intersect, but then these intersections are also components of $S(0)$.

Each snapshot $S_{j}$ is then discretized (with arbitrary accuracy) by a finite mesh of points $X_{j}=\left\{x_{1}^{j}, \cdots, x_{N_{j}}^{j}\right\}$ where typically the number $N_{0}$ of points on the reference configuration $S_{0}$ is much smaller than the number $N_{j}, 1 \leqslant j \leqslant q$, of points on the other given snapshots. Indeed, the complexity of the numerical problem to be solved after discretization is essentially determined by the number $N_{0}$ of discrete trajectories recomputed at each step of each inner iteration.

In the medical imaging applications below, each snapshot component had actually been previously modeled $[7,8]$ by NURBS equations fitted to image data. For each such snapshot components (curve segment or open surface patch with piecewise continuous boundary), the NURBS model can easily be used to generate point meshes approximating this component with arbitrary accuracy. For surfaces with boundaries, we separately generate two approximating meshes, one for the surface interior and one for the boundary; the adequate number of points on each one of these two sub-meshes is determined by fixing the same uniform approximation accuracy for the interior surface and for the boundary.

Multiscale methods are an important tool in concrete applications of our approach to multiple snapshots matching for deformable shapes observed in 3D-medical image sequences. Typically multi-scaling implies the use of increasingly finer finite point meshes to discretize 
the given multiple snapshots and the initial reference $S(0)$. The numerical solution computed as above at a given approximation scale then becomes the initialization of the gradient descent for the next finer scale. We have not presented numerical results on multiscaling, essentially because the human mitral valve deformations studied below concern a complex but fairly small 3D-deformable shape of diameter less than $35 \mathrm{~mm}$, acquired by live 3D-imaging devices with a spatial resolution of the order of $0.5 \mathrm{~mm}$. Several important implementation issues are discussed below. The initialization of the numerical gradient descent is outlined in 5.2.1. The Hausdorff distance matching term (3.4) plays an efficient part in our numerical implementations, but must be smoothed (see 5.2.2 ) to avoid using generalized gradients for nonsmooth functions. The choice of the scale parameter $\sigma$ for the radial Gaussian kernels (see 5.2.4) has a strong impact on the spatial smoothness of deformations and must roughly match the accuracy of the discretization meshes.

5.2.1. Initialization of the Gradient Method with Armijo Line Search. We first note that after the time and space discretization in order to initialize the unknown vector $\boldsymbol{\alpha}$ defined above in 5.1, one can initialize first the unknown family of time dependent vector fields $v=\left(v_{t}(x)\right)$, and then invert, for each value $\mathrm{t}$ of the discretized times, a large linear system of the type $v=M_{t} \alpha$, where $M_{t}$ is a positive definite matrix for which the non zero coefficients are of the form $K(x, y)$ for various $x, y \in R^{3}$. Here, $K$ is the fixed radial Gaussian kernel defining the kinetic energy.

Of course to avoid this initial inversion of large matrices, one can crudely initialize $\boldsymbol{\alpha}$ by setting $\boldsymbol{\alpha}=0$. This turns out to be an acceptable choice in several of our numerical applications, but clearly does not allow the use of existing complementary information on the solution which may be known in concrete situations.

Rough initialization by a smooth flow of affine transformations. Given two snapshots $S$ and $S^{\prime}$ of homeomorphic 3D-surfaces with boundaries, one can discretize $S$ and $S^{\prime}$ by two finite point meshes $X$ and $X^{\prime}$. Diagonalization of the inertia matrices of $X$ and $X^{\prime}$ around their centers of gravity $O, O^{\prime}$ generates the unit eigenvectors $e_{1}, e_{2}, e_{3}$ and $e_{1}^{\prime}, e_{2}^{\prime}, e_{3}^{\prime}$ with associated positive eigenvalues $\gamma_{1}, \gamma_{2}, \gamma_{3}$ and $\gamma_{1}^{\prime}, \gamma_{2}^{\prime}, \gamma_{3}^{\prime}$. Call $T$ the translation mapping $O$ on $O^{\prime}, R$ the rotation mapping $e_{1}, e_{2}, e_{3}$ on $e_{1}^{\prime}, e_{2}^{\prime}, e_{3}^{\prime}$, and $A$ the affinity mapping $e_{1}, e_{2}, e_{3}$ on $\frac{\gamma_{1}^{\prime}}{\gamma_{1}} e_{1}^{\prime}, \frac{\gamma_{2}^{\prime}}{\gamma_{2}} e_{2}^{\prime}, \frac{\gamma_{3}^{\prime}}{\gamma_{3}} e_{3}^{\prime}$. The affine linear transformation $L=A R T$ of $R^{3}$ maps $X$ on $L X$, which has the same matrix of inertia as $X^{\prime}$. One can obviously imbed explicitly and separately $A, R, T$ into differentiable semi-groups $A(t), R(t), T(t)$ of affinities, rotations, and translations, such that $[A(1), R(1), T(1)]=[A, R, T]$ and $[A(0), R(0), T(0)]=[I d, I d, I d]$, where $I d$ is the identity transformation of $R^{3}$. Then the affine linear transformations $L(t)=A(t) R(t) T(t)$ are $R^{3}$-diffeomorphisms depending smoothly on $t$ such that $L(0)=I d, L(1)=L$. They provide a first rough initialization for the unknown flow of diffeomorphisms matching $X$ and $X^{\prime}$. The associated vector fields defined for $t \in R^{+}, \quad x \in R^{3}$ by $v_{t}(x)=\frac{d L(t)}{d t} x$ are a crude initialization for the numerical search of a vector field solution of an optimal matching between $S$ and $S^{\prime}$.

For the multiple snapshots case, one applies this initialization successively between $S_{j}$ and $S_{j+1}$ on the time interval $\left[t_{j}, t_{j+1}\right]$ to compute an initial family of time dependent vector fields $v_{t}(x)$ such that the associated diffeomorphic flow quite roughly matches $S_{0}$ with the successive snapshots $S_{j}$.

Hierarchical initialization by B-splines matching. Consider two homeomorphic bounded 3D-surfaces $S_{0}$ and $S_{1}$ discretized by finite point meshes $X_{0}, X_{1}$ with compact piecewise smooth boundaries $B_{0}$ and $B_{1}$. Select arbitrary arc length origins $P_{0}$ and $P_{1}$ on $B_{0}$ and 
$B_{1}$ and let $\left(a_{0}, a_{1}\right)$ be the corresponding Euclidean arc lengths abscissas on $B_{0}$ and $B_{1}$. Define a piecewise smooth diffeomorphism $f$ of $B_{0}$ onto $B_{1}$ as follows: for each $Q \in B_{0}$, set $f(Q)=U \in B_{1}$ where $a_{1}(U)=c a_{0}(Q)$ and $c$ is the ratio of the lengths of $B_{0}$ and $B_{1}$. Using the gradient descent algorithm defined above, we can then numerically determine a time dependent vector field $\left.w=w_{t}(x)\right), t>0, x \in R^{3}$ such that the associated flow of $R^{3}$ diffeomorphisms $F_{t}$ verifies $F_{1}(Q)$ close to $f(Q)$ for all $Q \in B_{0}$. This is an easy optimization problem, since $B_{0}$ is one dimensional and the desired mapping $f$ of $B_{0}$ onto $B_{1}$ is known, so that numerical convergence is quite fast.

One can then generate a discretized surface $X_{\tau}=F_{\tau}\left(X_{0}\right)$ with boundaries $B_{\tau}=F_{\tau}\left(B_{0}\right)$ for any $\tau \leqslant 1$. Select a $\tau<1$ fairly close to 1 and discretize $B_{\tau}$ by a finite mesh $\partial X_{\tau}$. Fix $\varepsilon>0$. By a relaxation algorithm, provided $\varepsilon$ is not too small, it is possible to select targets $z(x) \in B_{1}$ for each $x \in X_{0}$ such that the map $x \rightarrow z(x)$ is injective on $X_{0}$, and such that the points $z(x)$ and $F_{\tau}(x)$ verify

$$
\left\|z(x)-F_{\tau}(x)\right\|<\varepsilon+d\left(F_{\tau}(x), X_{1}\right), \quad \text { where } \quad d\left(y, X_{1}\right)=\min _{u \in X_{1}}\|u-y\| .
$$

We then seek an $R^{3}$-valued function $\operatorname{Pol}(t, x)$ defined for $\tau \leqslant t \leqslant 1$ and $x \in R^{3}$ by linear combinations of cubic B-splines and such that we have $\operatorname{Pol}(\tau, x)=x$ for all $x \in X_{\tau} \cup \partial X_{\tau}$, and $\operatorname{Pol}(1, x)=z(x) \forall x \in X_{\tau}$. This involves the resolution of a standard linear system for B-splines. Our initial time dependent vector fields $v=v_{t}(x)$ for gradient descent is then defined by $v_{t}=w_{t}$ for $0 \leqslant t \leqslant \tau$ and by $v_{t}(x)=\partial_{t} \operatorname{Pol}(t, x)$ for all $x \in R^{3}$ and $\tau<t \leqslant 1$.

5.2.2. Smoothing of the Hausdorff Disparity. In general, the Hausdorff distance (3.4) is not a smooth function. For our gradient descent with Armijo line search we define a smoothed version of the Hausdorff disparity as follows. Consider two compact 3D-shapes $S$ and $S^{\prime}$ discretized by finite meshes $X$ and $Y$. Define functions $\phi: X \rightarrow Y$ and $\psi: Y \rightarrow X$

$$
\begin{gathered}
\phi(x)=\operatorname{argmin}_{y \in Y}\|x-y\| \quad \forall x \in X, \\
\psi(y)=\operatorname{argmin}_{x \in X}\|y-x\| \quad \forall y \in Y .
\end{gathered}
$$

Clearly, these two functions are continuous but not necessarily injective. For each $x \in X$ define $U(x) \subset X$ as the set of the $r$ closest neighbors of $x$ in $X$ including $x$. Similarly, one defines neighborhoods $U(y) \subset Y$ of $y$ for all $y \in Y$. The smoothed Hausdorff disparities $h(X, Y)$ and $h(Y, X)$ are defined by

$$
\begin{gathered}
h(X, Y)=(1 / r|X|) \sum_{x \in X} \sum_{z \in U(\phi(x))}\|x-z\|^{2}, \\
h(Y, X)=(1 / r|Y|) \sum_{y \in Y} \sum_{z \in U(\psi(y))}\|y-z\|^{2},
\end{gathered}
$$

where $|X|,|Y|$ are the cardinals of $X, Y$. The numerical gradients of $h(X, Y)$ and $h(Y, X)$ with respect to variations of $X$ when $Y$ remains fixed are always approximated by "freezing" temporarily the points $\phi(x)$ and $\psi(y)$, since the functions $\phi$ and $\psi$ are not everywhere differentiable.

The smoothed Hausdorff disparity between $X$ and $Y$ will be defined by $D_{H}(X, Y)=$ $h(X, Y)+h(Y, X)$, and will sometimes be referred to below as "global Hausdorff disparity" between $X$ and $Y$. For diffeomorphic matching of surfaces $S$ and $S^{\prime}$ with boundaries $\partial S$ and $\partial S^{\prime}$, we always compute separate Hausdorff disparities between discretized versions of the interior sets $S^{o}, S^{\prime o}$ and of the boundaries $\partial S, \partial S^{\prime}$. 
5.2.3. Choice of the Scale Parameters in the Gaussian Kernels. When the scale parameter $\sigma>0$ of the radial Gaussian kernel $K_{\sigma}$ (3.3) defining the Hilbert space $V=V_{K}$ increases, then the spatial smoothness of the optimal diffeomorphic deformations tends to increase. Call $X$ the finite discretization of the interior of an initial surface or curve $S_{0}$. Define $\operatorname{dim}(X)$ as the dimension of $S_{0}$. For each $x \in X$, and each fixed integer $r>0$, define as above $U(x) \subset X$ as the set of the $r$ closest neighbors of $x$ in $X$, including $x$ itself. We then define the radius function $\rho(x)>0$ by

$$
\rho(x)=\max _{z \in U(x)}\|x-z\| \forall x \in X .
$$

The maximum $R(X)=\max _{x \in X}(\rho(x))$ of the function $\rho$ concretely defines the local mesh size of $X$.

We naturally chose $r=3$ when $S_{0}$ is a curve segment or when $X$ is a finite discretization of the boundary $\partial S_{0}$, and $r=5$ when $S 0$ is a surface.

Based on the local mesh size $R(X)$ of $X$, we select the scale parameter $\sigma$ for the radial Gaussian kernel defining the kinetic energy as follows

$$
\sigma=\kappa 2^{-1 / 2} R(X)
$$

where $\kappa$ is some constant satisfying $2 \geqslant \kappa \geqslant 1$. This choice clearly bounds the number of neighbors $y \in X$ of any given point $x \in X$ such that the deformation trajectory of $y$ influences the deformation trajectory of $x$.

The scale parameter $\sigma$ is generally kept fixed during the whole gradient descent, but can be updated dynamically after enough iterations. Numerical evidence suggests to choose $\sigma$ according to (5.20) and to keep it fixed during the whole process, when there is not much difference between the local mesh sizes selected for the multiple snapshots.

The scale parameter of the radial Gaussian kernel defining the Hilbert distance between bounded measures on $R^{3}$ (see (3.6) ) is selected to have the order of magnitude of the Hausdorff distances between the given snapshots $S_{j}$ and the current deformed shapes $\hat{S}_{j}$ at instants $t_{j}$ and is updated periodically, in particular when the initialization is not close enough to the assigned multiple snapshots.

5.2.4. Continuation in the Regularization Parameter. Consider first the situation where the weights $\lambda_{j}$ in the disparity cost functional functional (3.7) are all equal to the same $\lambda>0$. This regularization parameter provides a weighting between the kinetic energy (3.1) and the disparity cost functional. For $\lambda$ small, the regularizing effect of the kinetic energy dominates, whereas large values of $\lambda$ enhance the matching quality of deformed shapes $\hat{S}_{j}$ and the given snapshots $S_{j}$. To reach a good matching quality, one needs to minimize the objective function $J$ for fixed but sufficiently large $\lambda$. However, for increasing $\lambda$, the system of optimality equations equivalent to grad $J=0$ becomes more and more ill-conditioned, which may result in divergence of the gradient descent. A convenient remedy to overcome this obstacle is to use an appropriate "continuation" in $\lambda$. The continuation method consists in combining outer iterations in $\lambda$ with inner iterations at fixed $\lambda$, which we chose to implement by classical gradient descent with Armijo line search (cf., e.g., [2]).

To monitor progress during the outer iterations, we compute $q$ performance indicators Dist $_{j}$, one for each given snapshot $S_{j}, j=1, \cdots, q$, as follows:

$$
j=1, \cdots, q, \quad \text { Dist }_{j}:=90 \text { th percentile of }\left\{d_{1}^{j}, \cdots, d_{N_{0}}^{j}\right\},
$$


where the distances $d_{n}^{j}, n=1, \cdots, N_{0}$, are defined by

$$
d_{n}^{j}=\min _{m=1, \cdots, N_{j}}\left\|x_{n}\left(t_{j}\right)-x_{m}^{j}\right\|
$$

Hence, each Dist ${ }_{j}$ provides an upper bound for $90 \%$ of the current geometric errors affecting the points of the current deformed surface $\hat{S}_{j}$. In practical applications, the given snapshots $S_{j}$ are typically determined by 3D-image data where geometric accuracy is bounded by the image resolution. Algorithmic modeling of the $S_{j}$ by NURBS for instance also introduces other sources of geometric inaccuracy in the $S_{j}$ data. Hence one can generally preassign a target threshold level THR $>0$ for the performance indicators Dist ${ }_{j}$, and we can say that good matching with all the intermediary snapshots has been reached as soon as all the Dist ${ }_{j}$ are inferior to THR.

As described above, the continuation method implements a succession of inner iterations, which are gradient descents at fixed $\lambda$, and at the end of each inner iteration,the regularization parameter $\lambda$ is increased (outer iteration) by a constant multiplicative factor $\gamma>1$.

At the end of each inner iteration, we want the norm of gradient $J$ to have decreased at least by a fixed multiplicative factor $\theta<1$.

Formally the continuation method reads as follows:

\section{Step 1 (Initialization of the outer iteration)}

Specify a small initial value $\lambda_{0}>0$ and set $\nu=0$.

Step 2 (Initialization of the inner iteration)

Compute $\boldsymbol{\alpha}_{\nu}^{(0)}$ by one of the initialization procedures as outlined above in subsection 6.1 and set $\mu=0$.

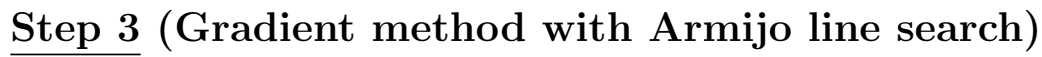

Step 3.1 Set $\mu:=\mu+1$ and compute $\boldsymbol{\alpha}_{\nu}^{(\mu)}$ by gradient descent with Armijo line search.

Step 3.2 If the gradient $\nabla J$ of the objective function $J$ verifies the termination criterion

$$
\mid \nabla J\left(\boldsymbol { \alpha } _ { \nu } ^ { ( \mu ) } | \leqslant \theta | \nabla J \left(\boldsymbol{\alpha}_{\nu}^{(0)} \mid\right.\right.
$$

is satisfied, go to Step 4. Otherwise, go to Step 3.1.

If the gradient method fails to converge, adjust the parameter in the Armijo line search, set $\mu:=0$, and go to Step 3.1.

\section{Step 4 (Termination of the outer iteration)}

If the threshold based termination criterion

$$
\text { Dist }_{j}<\mathrm{THR}, \quad 1 \leqslant j \leqslant q,
$$

is satisfied, stop the algorithm.

Otherwise, set $\nu:=\nu+1, \boldsymbol{\alpha}_{\nu}^{(0)}:=\boldsymbol{\alpha}_{\nu-1}^{(\mu)}$, and increase the regularization parameter by

$$
\lambda_{\nu}:=\gamma \lambda_{\nu-1}
$$

and go to Step 3 . 


\section{Numerical Results for the Human Mitral Valve Apparatus}

\subsection{Anatomy of the Mitral Valve}

In the cardiology application below, we use the previous variational techniques to numerically construct diffeomorphic flows roughly approximating the dynamic deformations between multiple 3D-snapshots of the human mitral valve apparatus. This apparatus is a biological valve integrated within the heart which has quasi-periodic dynamics tightly correlated to heartbeats enabling an essential periodic obturation of the blood flow to and from the heart. Our starting point is a patient specific finite set of $q+1$ static models $S_{j}$ of the mitral valve apparatus. These models were generated by image analysis of live 3D-echocardiographic movies at specific heart cycle instants $t_{j}$ in a recent study $[7,8]$ involving the Methodist Hospital, Houston, Texas (S. Ben Zekry, S. Little, W. Zoghbi, MDs) as well as a research group on mathematical image analysis led by R. Azencott at Univ. of Houston. Each 3D-echocardiographic movie includes twenty-seven to thirty 3D-frames per heartbeat cycle, acquired by ultrasound technology, and represents a high volume of image data corrupted by 'speckle' noise.

The mitral valve models $S_{j}$ are based on $N U R B S$ (non uniform rational B-splines), and were obtained in $[7,8]$ by combining optical flow extraction algorithms with sparse tagging by medical experts. The number $q+1$ of intermediary key heartbeat cycle instants $t_{j}$ ranged from 3 to 8 , and the time range $I=\left[t_{0}, t_{1}\right]$ covers either a half or a whole heartbeat cycle with total duration between $1 / 2$ to 1 second.

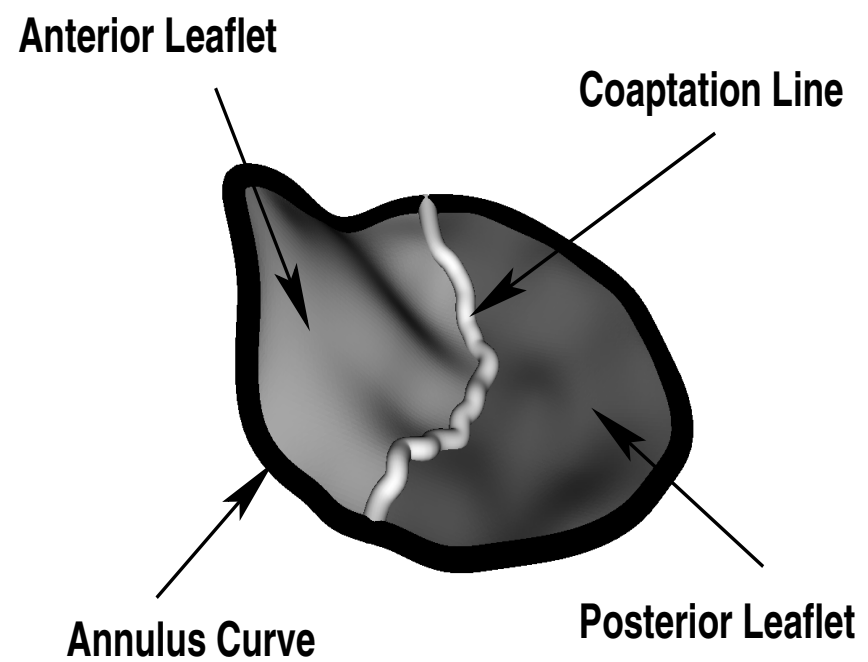

Fig. 6.1. Mitral valve: the middle line is the coaptation line along which the surfaces of the anterior and posterior leaflets meet when the valve is closed. The closed black thick curve is the mitral annulus

The mitral valve apparatus (MVA) involves the annulus (a closed thin deformable ring) and two deformable surfaces with boundaries, namely the anterior and posterior leaflets. These mitral leaflets are flexibly linked to the annulus by a subsegment of their boundaries. When the valve is closed, the exterior parts of the leaflets have a common boundary called the coaptation line.

The MVA can be viewed as a composite deformable object built from several smooth deformable shapes (see Fig. 6.1), namely a closed curve MA (the mitral annulus), a curve segment COA (the coaptation line), two surfaces AL and PL (the mitral leaflets) with 
boundaries $\partial \mathrm{AL}=\mathrm{COA} \cup$ antMA and $\partial \mathrm{PL}=\mathrm{COA} \cup$ postMA, where antMA and postMA are complementary subsegments of the MA.

\subsection{Multiple Snapshots for the Mitral Annulus and Leaflets}

The given snapshots are modeled by parametrized closed curves in $R^{3}$ for the mitral annulus, and by parametrized surfaces with boundaries in $R^{3}$ for the anterior and posterior leaflets. The parametric equations of these curve and surface models and of their boundaries have previously been extracted from 3D-image data as indicated above, and are explicit linear combinations of cubic B-splines which are tensor products of polynomials of degree 3 in one variable restricted to bounded intervals.

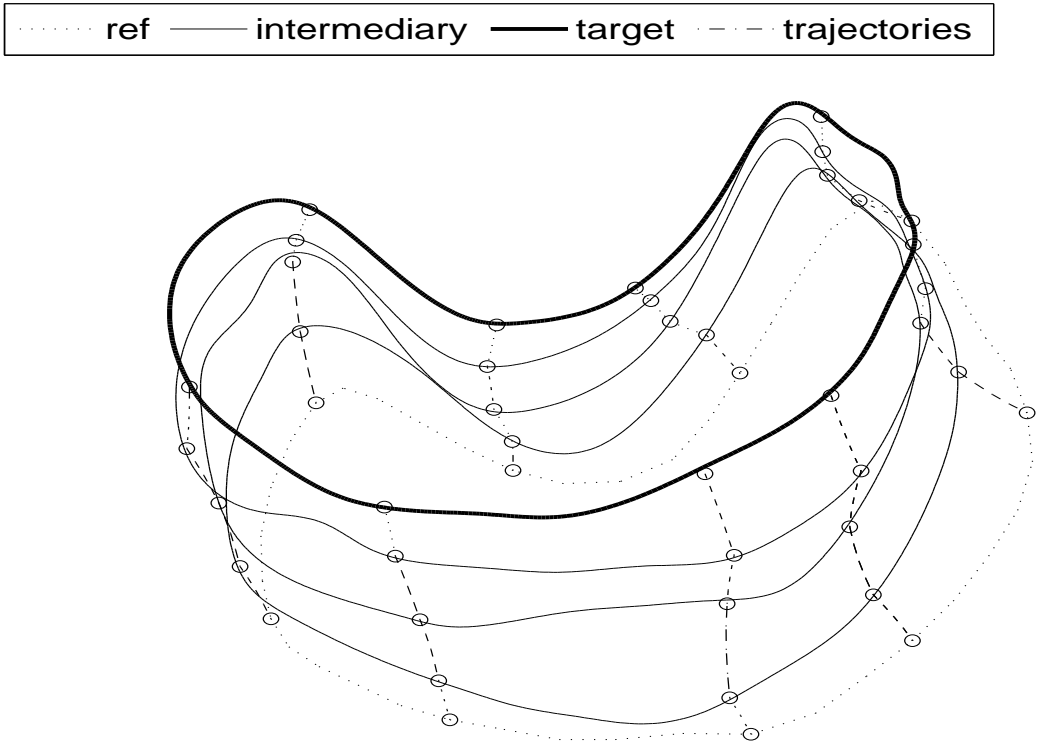

Fig. 6.2. The 5 closed curves are 5 successive annulus snapshots. The dotted curve is the initial snapshot. From bottom to top, the next 3 curves are the intermediary snapshots and the last one is the final target. The vertical '--' lines are computed deformation trajectories for selected points of the reference curve

For the annulus, we have 10 given snapshots, and we focus first on the 5 annulus snapshots $A_{0}, A_{1}, A_{2}, A_{3}, A_{4}$ corresponding to instants $t_{0}=1, t_{1}=3, t_{2}=5, t_{3}=7, t_{4}=10$. The time unit, equal to $1 / 27$ second, is the time interval between 2 frames.

The annulus B-spline models enable the selection of point meshes $X A_{j}$ on $A_{j}$ with equal arc length between successive points. The first discretizations used in the numerical implementations of continuation algorithms presented here start with 42 points for $X A_{0}$, and approximately 500 points for each one of the other $X A_{j}$.

For each mitral leaflet, 4 snapshots $S_{0}, S_{1}, S_{2}, S_{4}$ are available at instants $t_{0}=0, t_{1}=$ $1, t_{2}=5, t_{4}=10$. We discretize them by meshes for which distances between any mesh point and its closest neighbor are approximately constant. Separate discretizations are applied to the interior of the $S_{j}$ and to their boundaries. For the anterior leaflet, we initially select a mesh of 126 points on the reference surface $S_{0}$, split into 84 points for its interior and 42 points on its boundary, and meshes of approximately 1600 points each on each one of the other snapshots of the anterior leaflets. 


\subsection{Diffeomorphic Matching for Multiple Annulus Snapshots}

We sketch the results of optimized diffeomorphic matching for multiple annulus snapshots, first when the disparity term is the smooth Hausdorff disparity ("Hausdorff matching"), and second when the disparity is computed by Hilbert distances between Borel measures ("measure matching"). In both cases, we have used the continuation algorithm outlined above and the trivial initialization $\boldsymbol{\alpha}=0$ with discrete time steps $\Delta t^{\ell}=1 / 18$ and $\theta=0.3$ in the termination criterion (5.22) for gradient descent with Armijo line search. The scale parameter computed by (5.20) is $\sigma=3.53$ for the Gaussian kernel $K_{\sigma}$.

The computational performance of the continuation algorithm is evaluated first by the convergence history for the $q$ indicators of the geometric matching accuracy Dist $_{j}$ and for the corresponding values Disp $_{j}$ of the $q$ components of the disparity functional.

We also record and display the tradeoffs between these matching quality indicators and the kinetic energy of the corresponding deformation flows. At the end of each inner iteration of gradient descent with fixed regularization parameter $\lambda$, we generate a point on each one of the approximate Pareto frontiers displaying matching quality indicators as functions of the kinetic energy. For the global disparity term (sum of the $q$ individual snapshot disparities $D i s p_{j}$ ), we expect and empirically observe convexity of these approximate Pareto frontiers , viewed as usual as the location of the weak Pareto optima for the pair of competing criteria (kinetic energy versus matching disparity) (cf., e.g., [12]).

6.3.1. Smoothed Hausdorff Matching for Multiple Annulus Snapshots. The initial value $\lambda^{(0)}=1$ of the regularization parameter is increased at each outer iteration by the multiplicative factor $\gamma=1.1$ (cf. (5.24)). The convergence history is documented in Figure 6.3 which displays the decrease of the geometric accuracy indicators Dist $_{j}$ and of the disparity components $\operatorname{Disp}_{j}$ for our 4 snapshots $A_{j}, 1 \leqslant j \leqslant 4$. It takes 200 iterations to reach the threshold geometric accuracy required for this application (see (5.23)), but we have extended iterations beyond this value to obtain a more complete view of the Pareto frontiers.
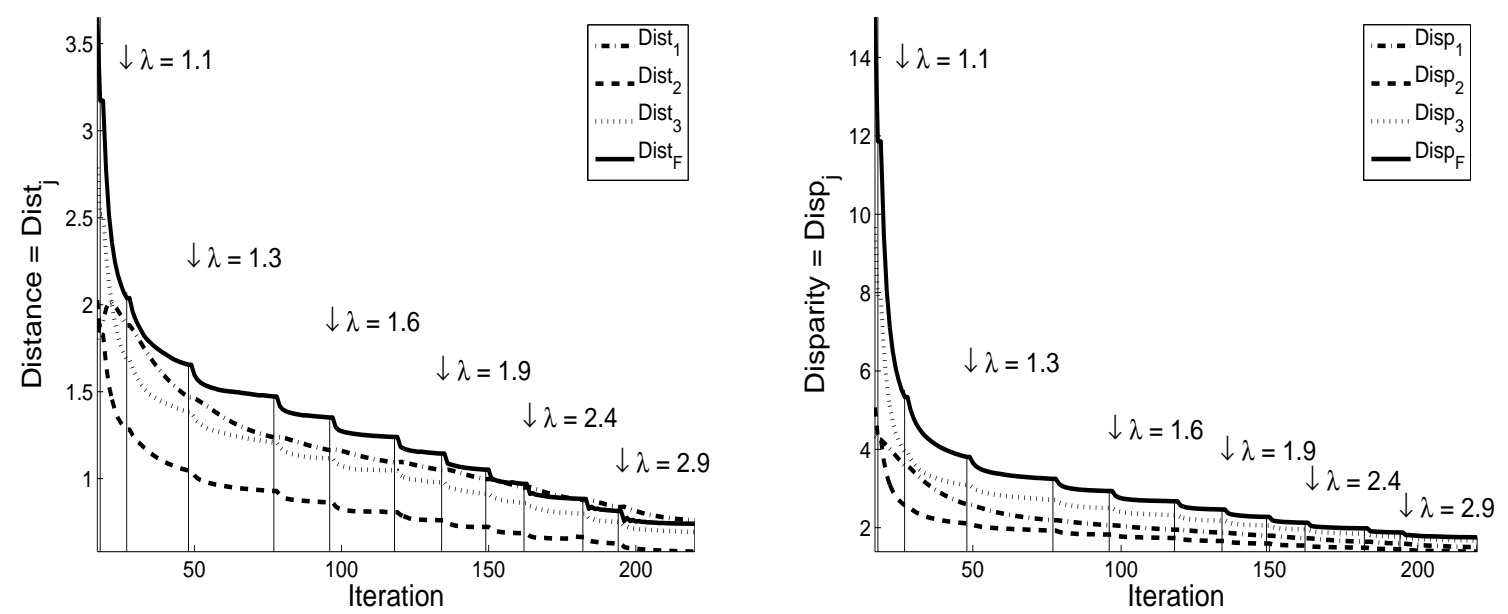

Fig. 6.3. Diffeomorphic matching for multiple mitral annulus snapshots, using smoothed Hausdorff distances: Convergence history for the geometric accuracy indicators Dist ${ }_{j}$ and for the smoothed Hausdorff disparity components Disp $_{j}$

The Pareto frontiers for the geometric accuracy indicators Dist ${ }_{j}$, the Hausdorff disparities $\operatorname{Disp}_{j}$, and the global Hausdorff disparity $\sum_{j} \operatorname{Disp}_{j}$ are shown in Fig. 6.4. Note that for the first annulus snapshot the geometric accuracy Dist ${ }_{1}$ and the Hausdorff disparity Disp d $_{1}$ do not 
exhibit a convex decrease pattern at the beginning of the continuation procedure. Indeed, the currently implemented algorithmic optimization strategy first "focuses" on matching the final snapshot $A_{4}$ and then successively shifts the focus on the matching of the intermediary snapshots $A_{3}, A_{2}, A_{1}$ in decreasing order. This is due to the fact that in the backwards adjoint ODE, the snapshots matching errors kick in successively in the same reverse order. Hence, as long as the final snapshots errors are large, the corrections implemented for the first snapshots remain quite fuzzy, and only turn out to be efficient once the matching errors on the final snapshots have become small enough.
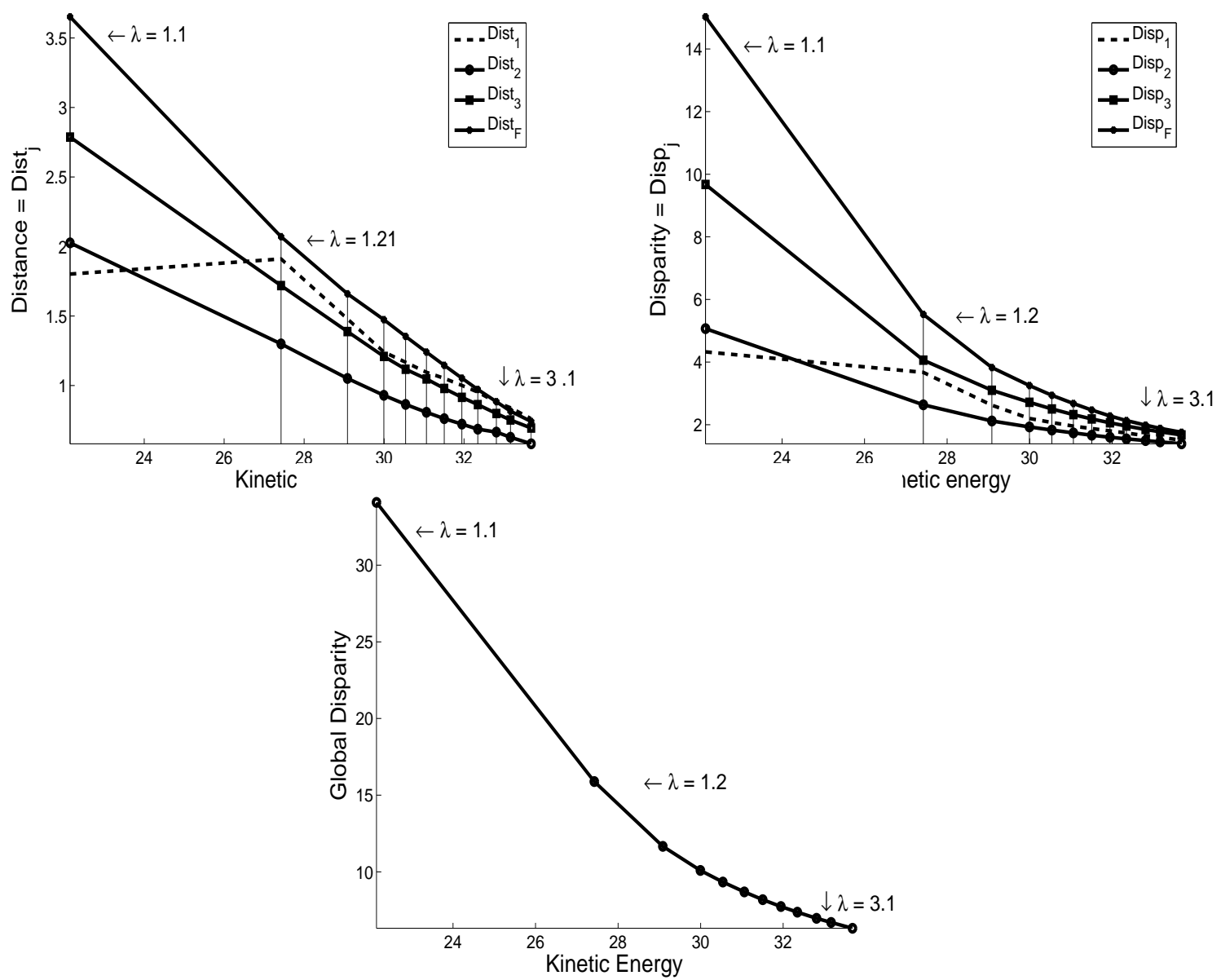

Fig. 6.4. Diffeomorphic matching for multiple annulus snapshots, using smoothed Hausdorff distances: Pareto frontiers for the geometric accuracy indicators Dist ${ }_{j}$ (top left), for the Hausdorff disparities $\operatorname{Disp}_{j}$ (top right), and for the global Hausdorff disparity $\sum_{j} \operatorname{Disp}_{j}$ (bottom)

\subsubsection{Diffeomorphic Matching for Multiple Annulus Snapshots: Measure}

Matching. Here, the disparity terms are the squared Hilbert distances between measures. The regularization parameter $\lambda$ starts at $\lambda^{(0)}=200$ and is multiplied by $\gamma=2$ at each outer iteration. Figure 6.5 displays the corresponding convergence history for 4 individual geometric accuracy indicators and 4 measure matching disparities. The desired threshold geometric accuracy is achieved for all snapshots after 70 iterations.

But the computing time for each iteration is about 3 times higher than for Hausdorff matching, due to the fact that measure matching invokes large numbers of evaluations of exponentials. This unfavorable computational feature of measure matching with respect to 
Hausdorff is quickly amplified when the number $N_{0}$ of trajectories increases.
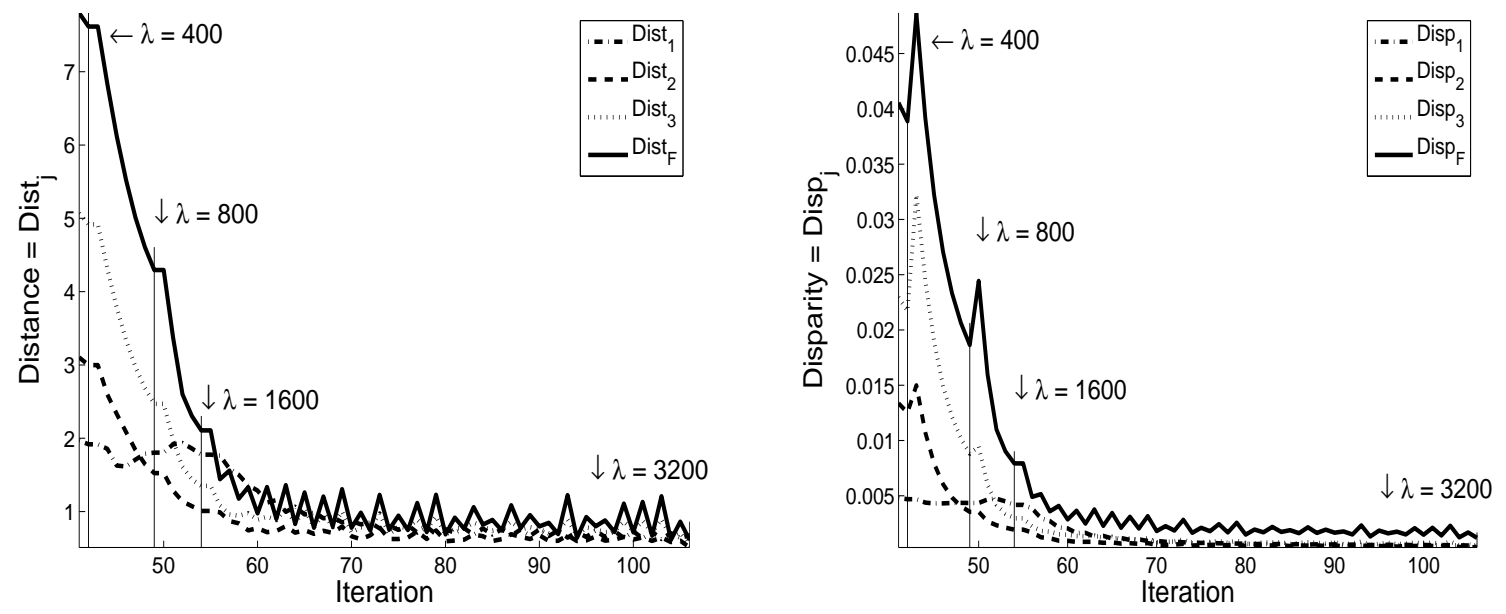

Fig. 6.5. Diffeomorphic matching for multiple annulus snapshots, using measure matching disparities: Convergence history for the individual geometric accuracy indicators Dist ${ }_{j}$ (left) and the measure matching disparities Disp $_{j}$ (right)

The corresponding Pareto frontiers are shown in Fig. 6.6. As in the case of Hausdorff matching, and for the same algorithmic reasons, the performance indicators improve first for the final snapshots and the improvements successively kick in for the other snapshots in reverse order of the snapshot times $t_{j}$. Comparing Fig. 6.6 and 6.4, we see that for a given geometric matching accuracy, the achievable kinetic energy remains higher for measure matching than for smoothed Hausdorff matching.

6.3.3. Diffeomorphic Matching for 10 Annulus Snapshots We consider here the 10 annulus snapshots $A_{j}, 0 \leqslant j \leqslant 10$, acquired at times $0,1,3,5,7,10,14,18,22,26$. We choose 42 points on the reference $A_{0}$ and approximately 500 points on the other snapshots. We use the smoothed Hausdorff disparity (3.4). We initialize $\boldsymbol{\alpha}$ by $\boldsymbol{\alpha}=0$, and use 52 discretized time steps. The parameters $\theta, \sigma, \rho$ are the same as above.

Since good matching accuracy for the first snapshots now takes a longer time (see Figure 6.7), we perform continuation with different weights $\lambda_{j}$ for the individual Hausdorff disparities Disp $_{j}$, adjusted to dynamically balance the current average sizes of these distinct disparities (cf. Fig. 6.8). Since we dynamically change the global disparity functional, we cannot expect to have nice convex Pareto frontiers ( cf. Fig. 6.8).

\subsection{Diffeomorphic Matching of the Anterior Leaflet}

We present the performances of diffeomorphic matching for 4 snapshots $S_{0}, S_{1}, S_{2}, S_{3}$ of the anterior leaflet, acquired at times 0,1,5,10, using smoothed Hausdorff snapshot disparities, where the disparities are separately computed for the boundary and the interior of each snapshot as outlined in 5.2.2. Indeed, initial experiments where this distinction was not implemented quickly displayed much poorer performances. Rough initial discretization starts with point meshes of cardinals 400 for $S_{0}$ and roughly equal to 1700 for $S_{1}$ and $S_{2}$.

Initialization for the time dependent vector field is implemented by piecewise constant vector fields for boundary deformations and adequately fitted polynomials in time and space variables for the interior of $S_{0}$, as sketched in subsection 5.1. The continuation algorithm 

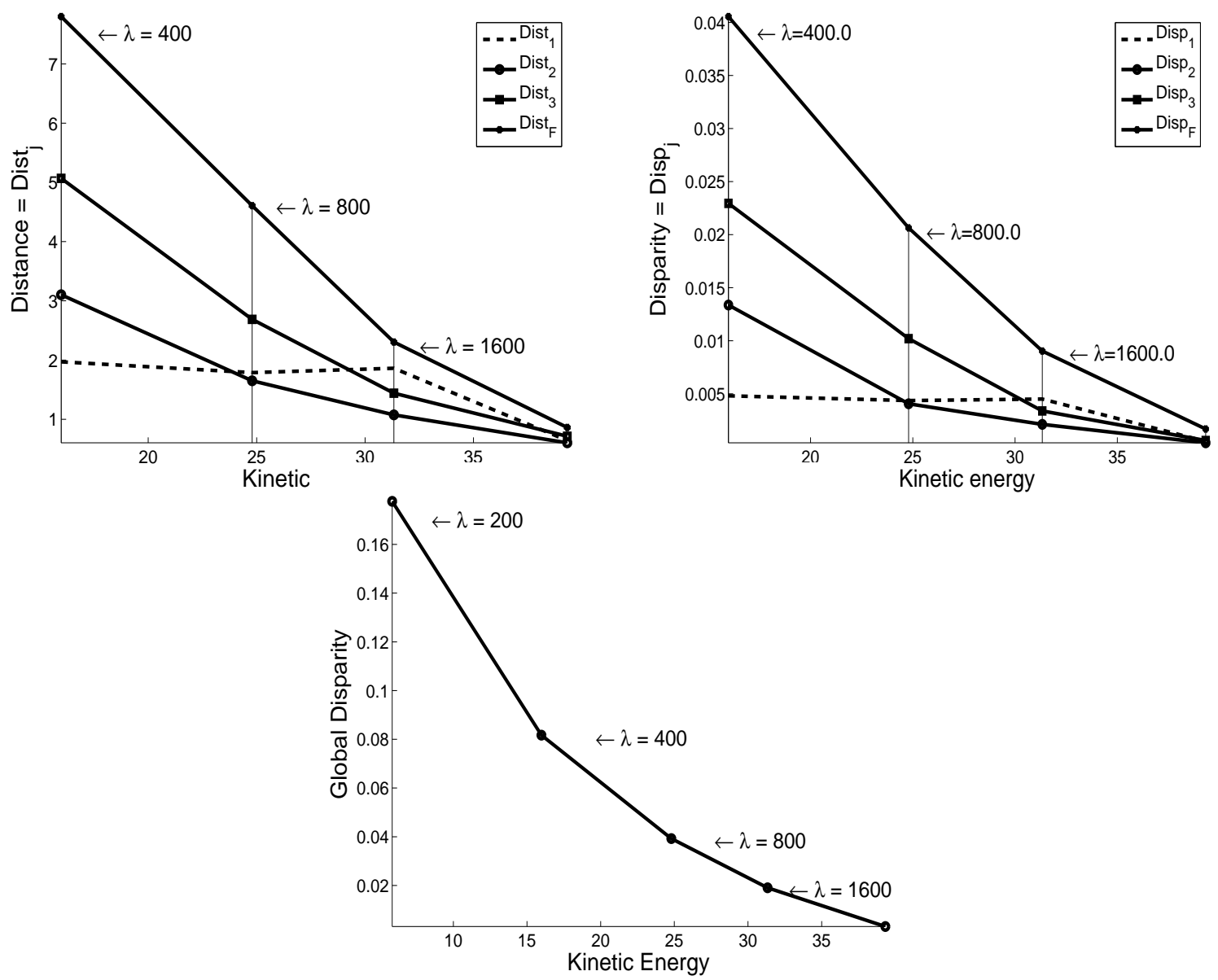

Fig. 6.6. Diffeomorphic matching for multiple annulus snapshots using measure matching disparities: Pareto frontiers for the geometric accuracy indicators Dist ${ }_{j}$ (top left), for the individual measure matching disparities $\operatorname{Disp}_{j}$ (top right), and for the global measure matching disparity $\sum_{j} \operatorname{Disp}_{j}$ (bottom)
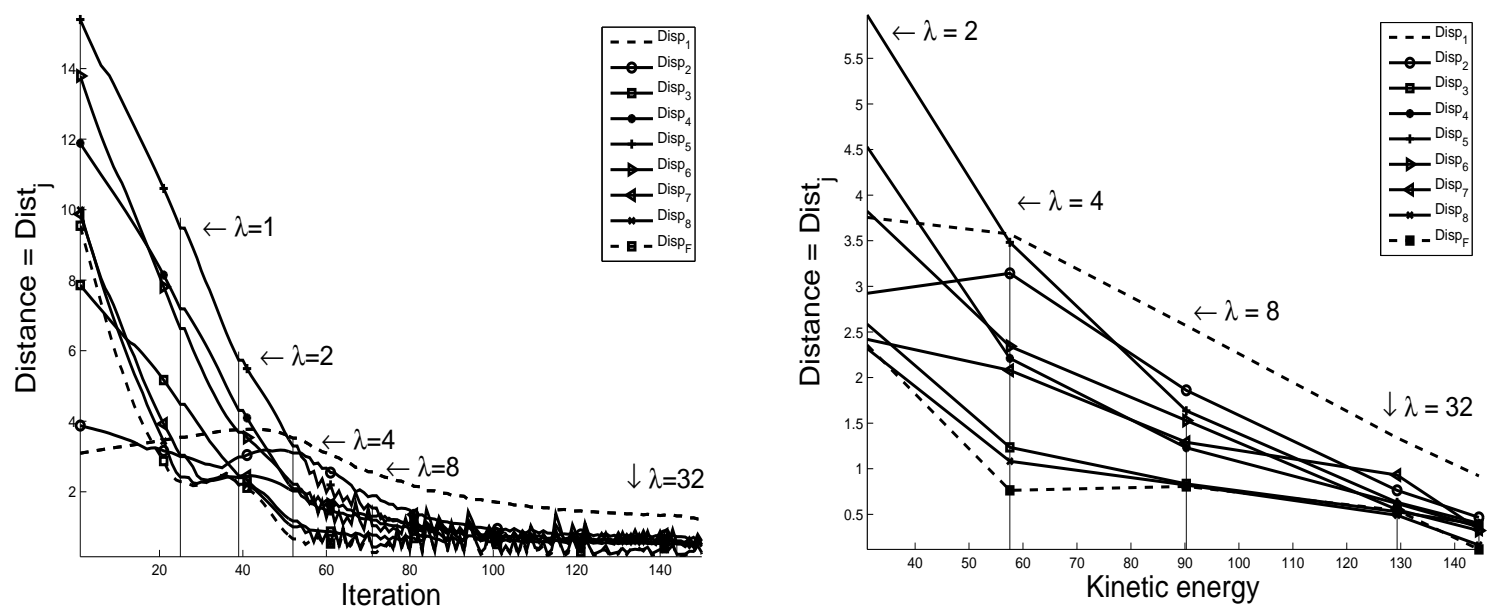

Fig. 6.7. Diffeomorphic matching for ten annulus snapshots using Hausdorff disparities: Convergence history of geometric accuracies (left) and corresponding Pareto frontiers (right) using a single regularization parameter $\lambda$ for the nine Hausdorff disparities 

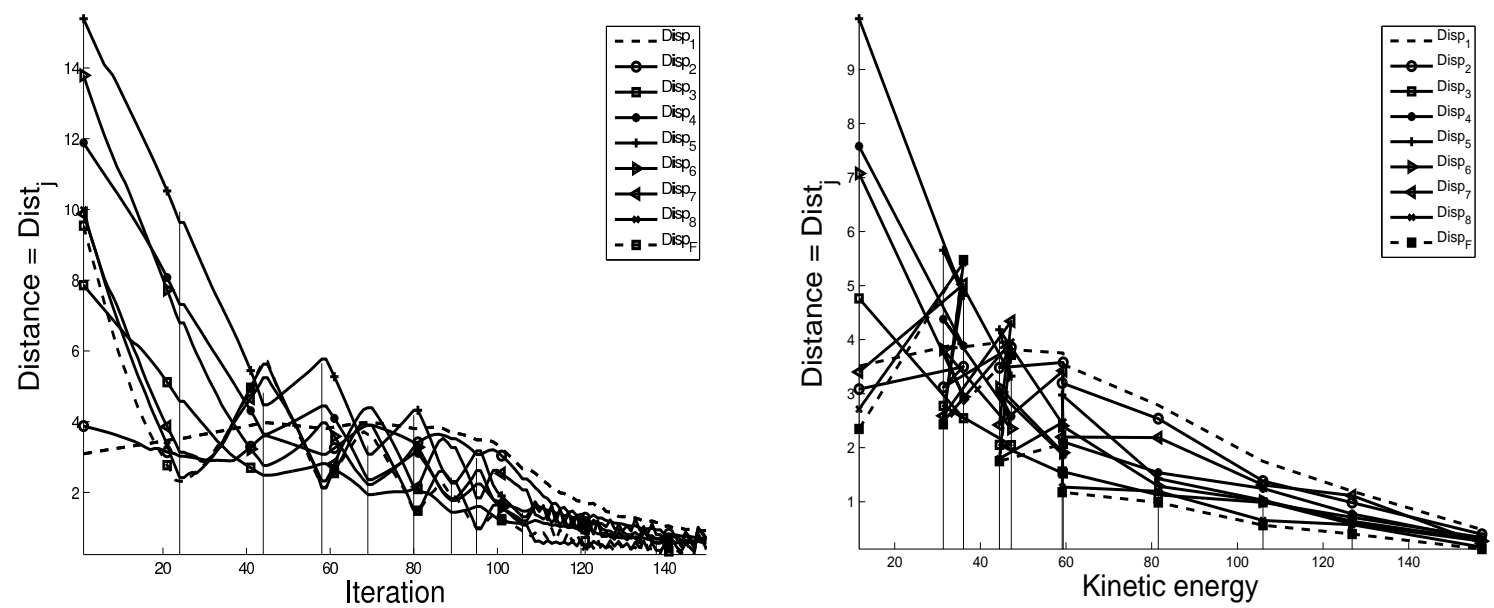

F i g. 6.8. Diffeomorphic matching for ten annulus snapshots: Convergence history of the geometric accuracies (left) and corresponding Pareto frontiers (right) using nine dynamic regularization parameters $\lambda_{j}$

starts with $\lambda=0.1$, and $\lambda$ is multiplied by $\gamma=1.5$ at each outer iteration. The scale and termination parameters $\sigma$ and $\theta$ are as above.

The following Fig. 6.9 and 6.10 display satisfactory performance results.
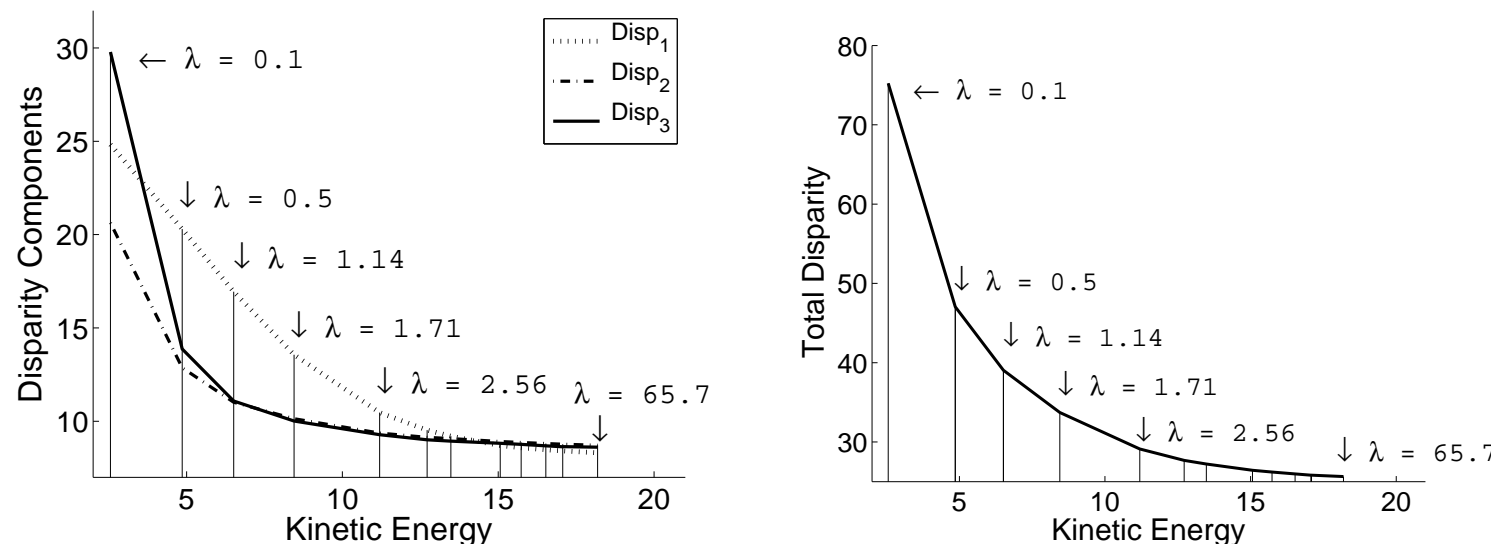

Fig. 6.9. Diffeomorphic matching of four anterior leaflet snapshots: Pareto frontiers for the separate Hausdorff disparities to snapshots (left) and for the global Hausdorff disparity (right)

We display in Fig. 6.11 a visualization of the computed deformations of the anterior leaflet at the 4 snapshot times $0,1,5,10$. The corresponding dynamic deformations of the anterior leaflet boundary are presented in Fig. 6.12. A few deformation trajectories are indicated. They are computed as solutions of the dynamics equations (3.2).

For each computed deformation $\hat{S}_{j}$ of the initial snapshot $S_{0}$, the three graphs in Figure 6.13 display several level curves for the point matching errors between $\hat{S}_{j}$ and the given anterior leaflets snapshots $S_{j}$. The coordinate system has been modified isometrically at each snapshot instant in order to display a better "horizontal" projection of $\hat{S}_{j}$. 

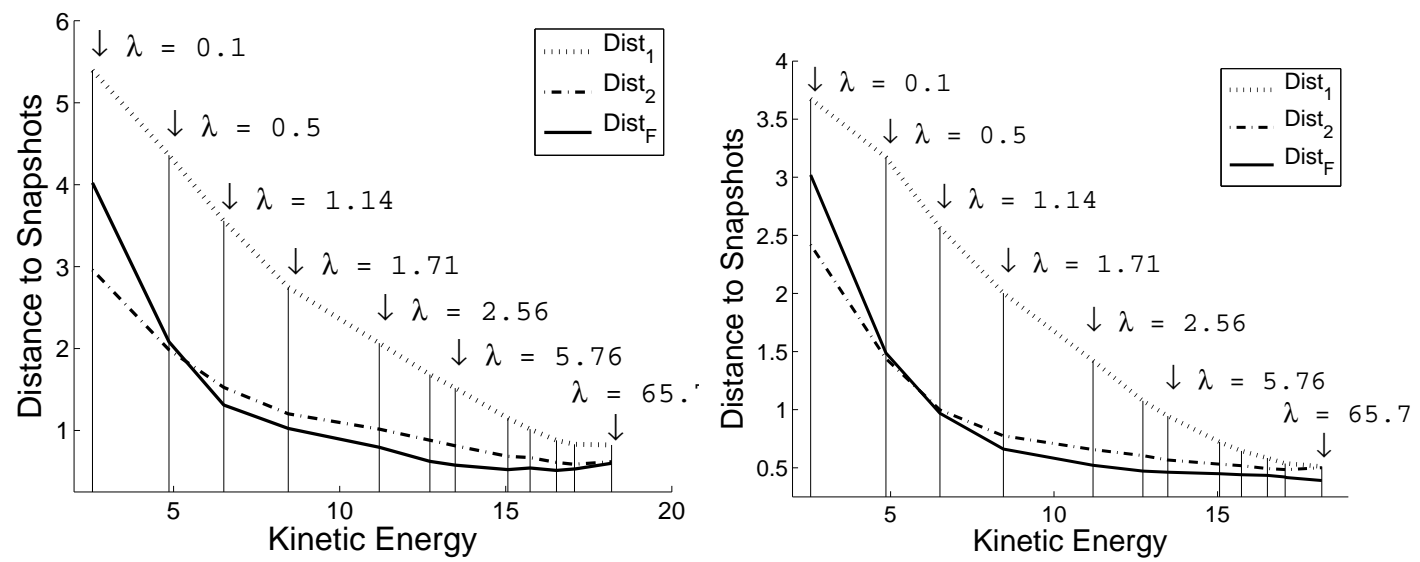

Fig. 6.10. Pareto frontiers for the maximum distances to snapshots (left) and for their 90th percentiles (right)
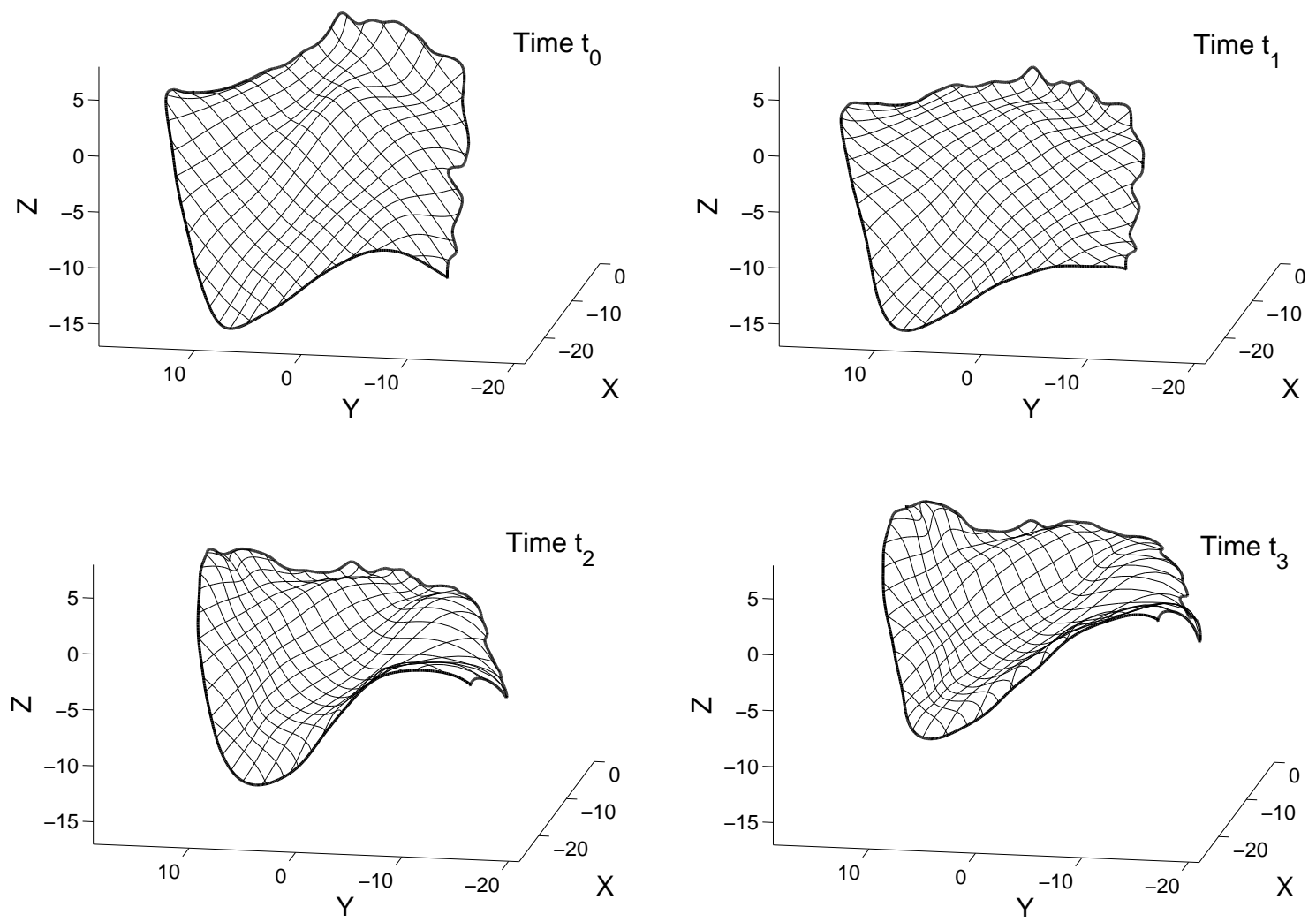

Fig. 6.11. Computed deformations matching four snapshots of the anterior leaflet at instants $0,1,5,10$ 

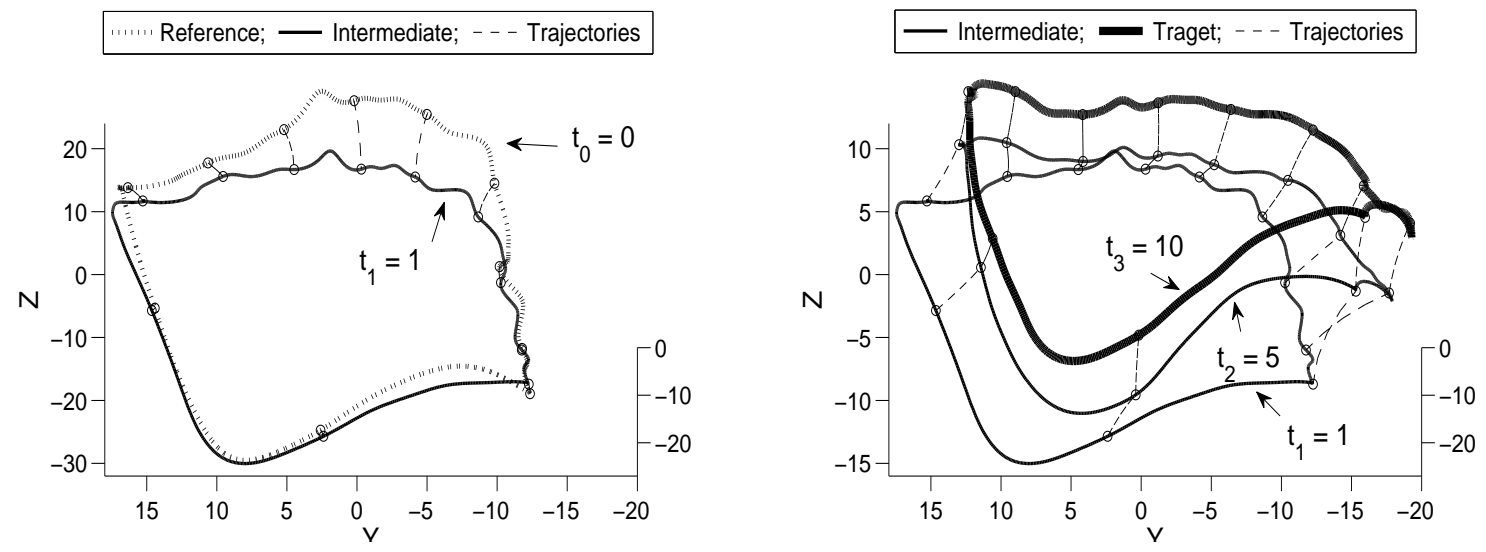

Fig. 6.12. Computed deformations of the anterior leaflet boundary: for easier visualization, the boundary deformations are displayed separately for instants 0,1 and for instants 1,5, 10
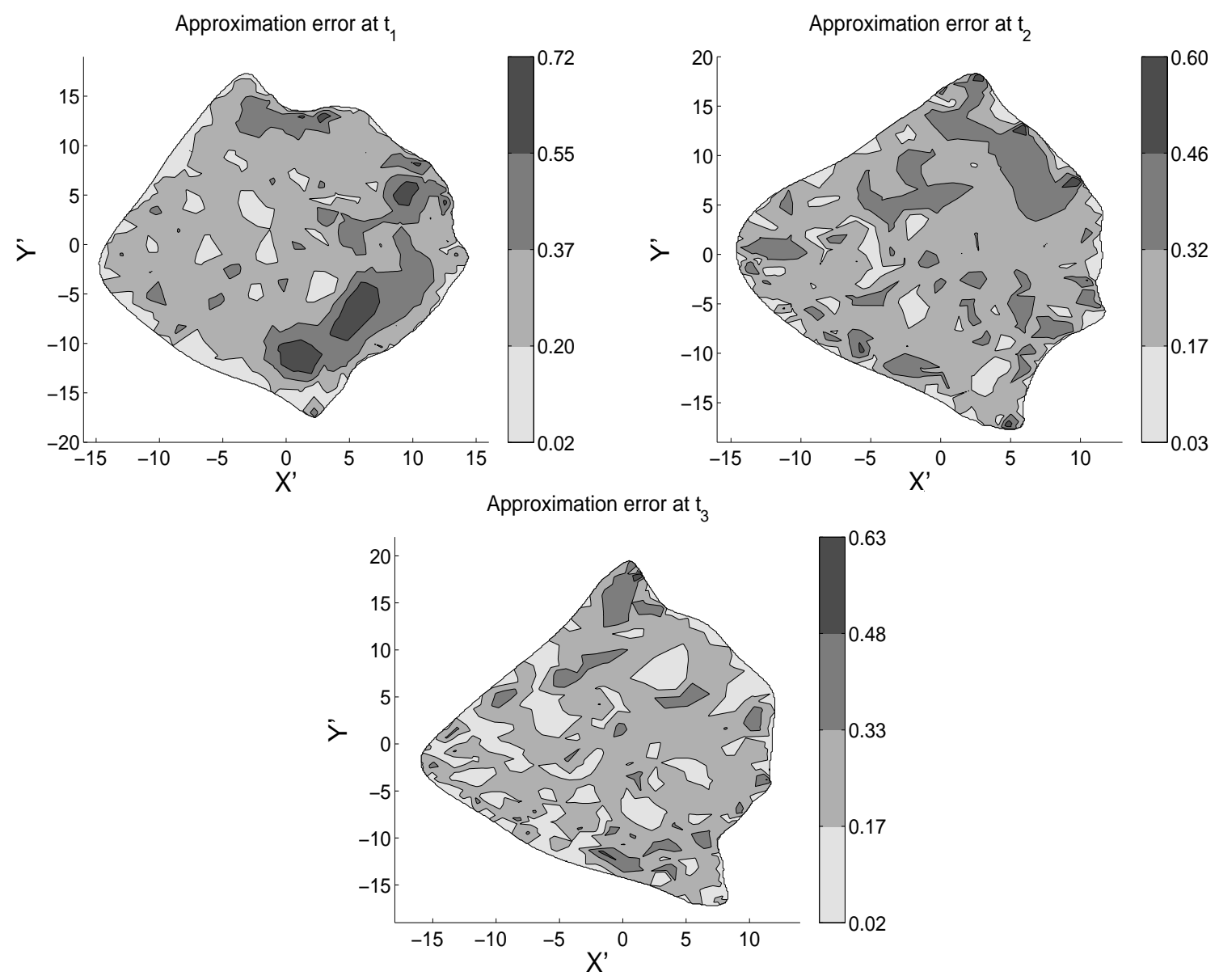

Fig. 6.13. Matching errors between the computed anterior leaflet deformations $\hat{S}_{j}$ and the snapshots $S_{j}$ 


\subsection{Diffeomorphic Matching for Multiple Snapshots of the Posterior Leaflet}

We have similarly implemented the diffeomorphic matching for 4 snapshots $S_{0}, S_{1}, S_{2}, S_{3}$ of the posterior leaflet acquired at times $0,1,5,10$, using smoothed Hausdorff snapshot disparities, with disparities separately computed for the surface boundary and the interior as outlined in 5.2.2. Time is discretized into 30 equal intervals. The 4 snapshots are discretized by point meshes of approximate cardinals 250 for the initial leaflet $S_{0}$, and 1100 for the other snapshots.

The initialization and the choice of the algorithmic parameters are similar to the implementation just described for the anterior leaflet (cf. 6.4). As already noted above, when one uses the same value $\lambda$ for all 3 regularization weights $\lambda_{j}$, the matching quality between $S_{j}$ and $\hat{S}_{j}$ improves more slowly for $j=1$ than for $j=3$ (see Fig. 6.14). We have compared this approach to a more adaptive one, where one dynamically adjusts the weights $\lambda_{j}$ at each outer iteration by appropriately balancing current values of the 3 Hausdorff disparities. (cf. Fig. 6.15 ). The geometric matching accuracies reach a desirable pragmatic threshold slightly faster for the dynamically independent weights, at the cost of some loss of convexity for the Pareto frontiers, which suggests that dynamic adjustment of weights may provide less robustness in the continuation procedure.
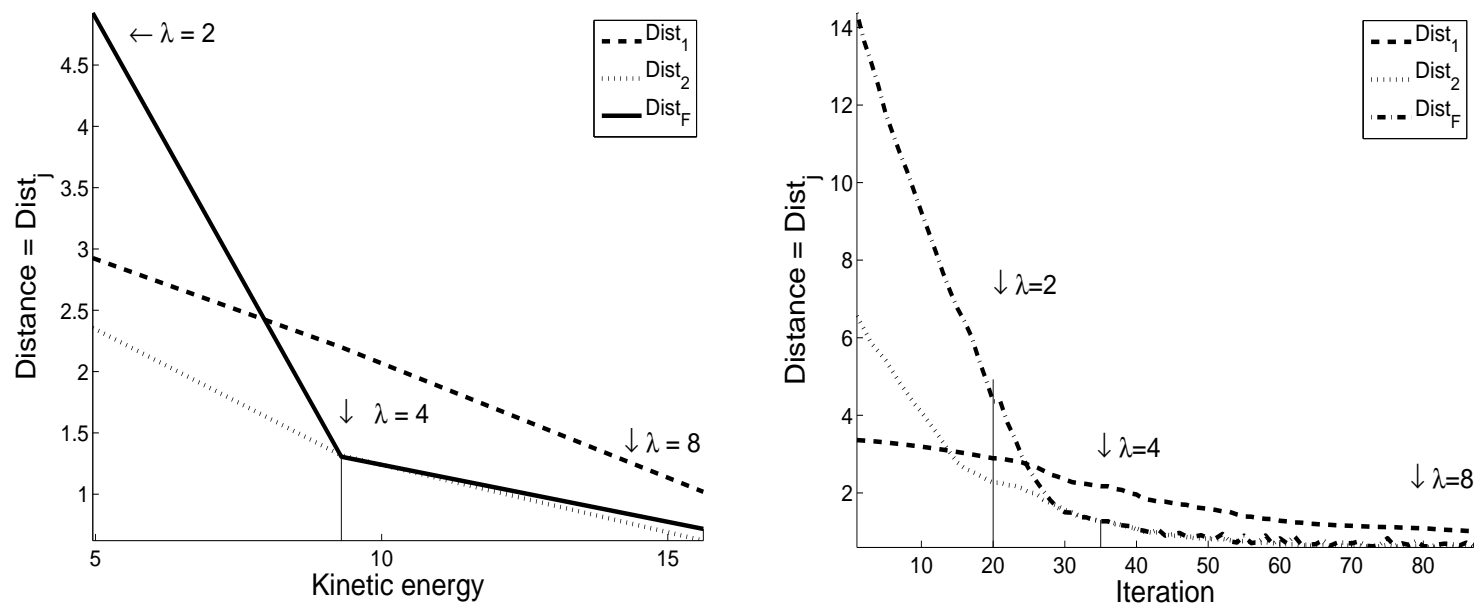

F ig. 6.14. Diffeomorphic matching for four posterior leaflet snapshots: Geometric accuracy indicators and Pareto frontiers for strictly equal regularization weights $\lambda_{j}$

Figure 6.16 displays the computed deformations of the posterior leaflet at the 4 instants $1,4,16,31$. The computed dynamic deformations of the posterior leaflet boundary are presented in Fig. 6.17 with a few deformation trajectories.

Figure 6.13 displays point matching errors between computed deformations $\hat{S}_{j}$ of the posterior leaflet and the given snapshots $S_{j}$. The coordinate system is modified isometrically for each computed deformation $\hat{S}_{j}$ to display a good "horizontal" projection of $\hat{S}_{j}$. 

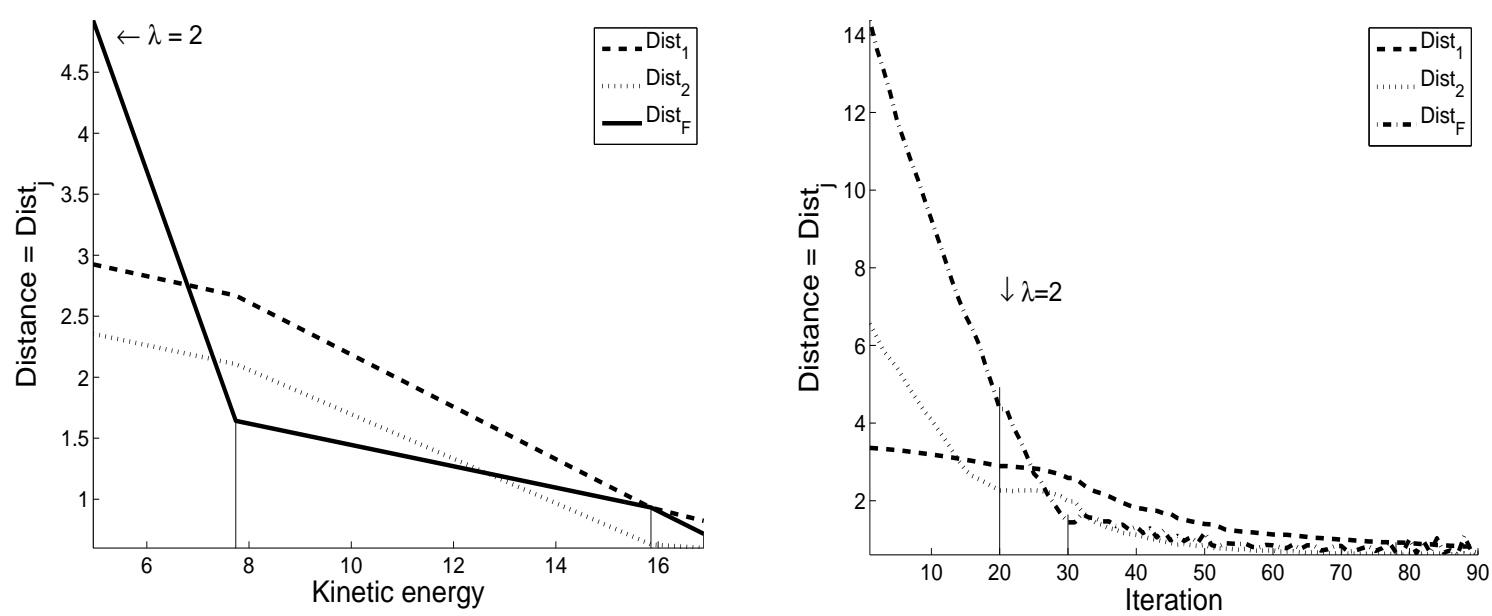

Fig. 6.15. Diffeomorphic matching for four posterior leaflet snapshots: Geometric accuracy indicators and Pareto frontiers for dynamically adjusted regularization weights $\lambda_{j}$
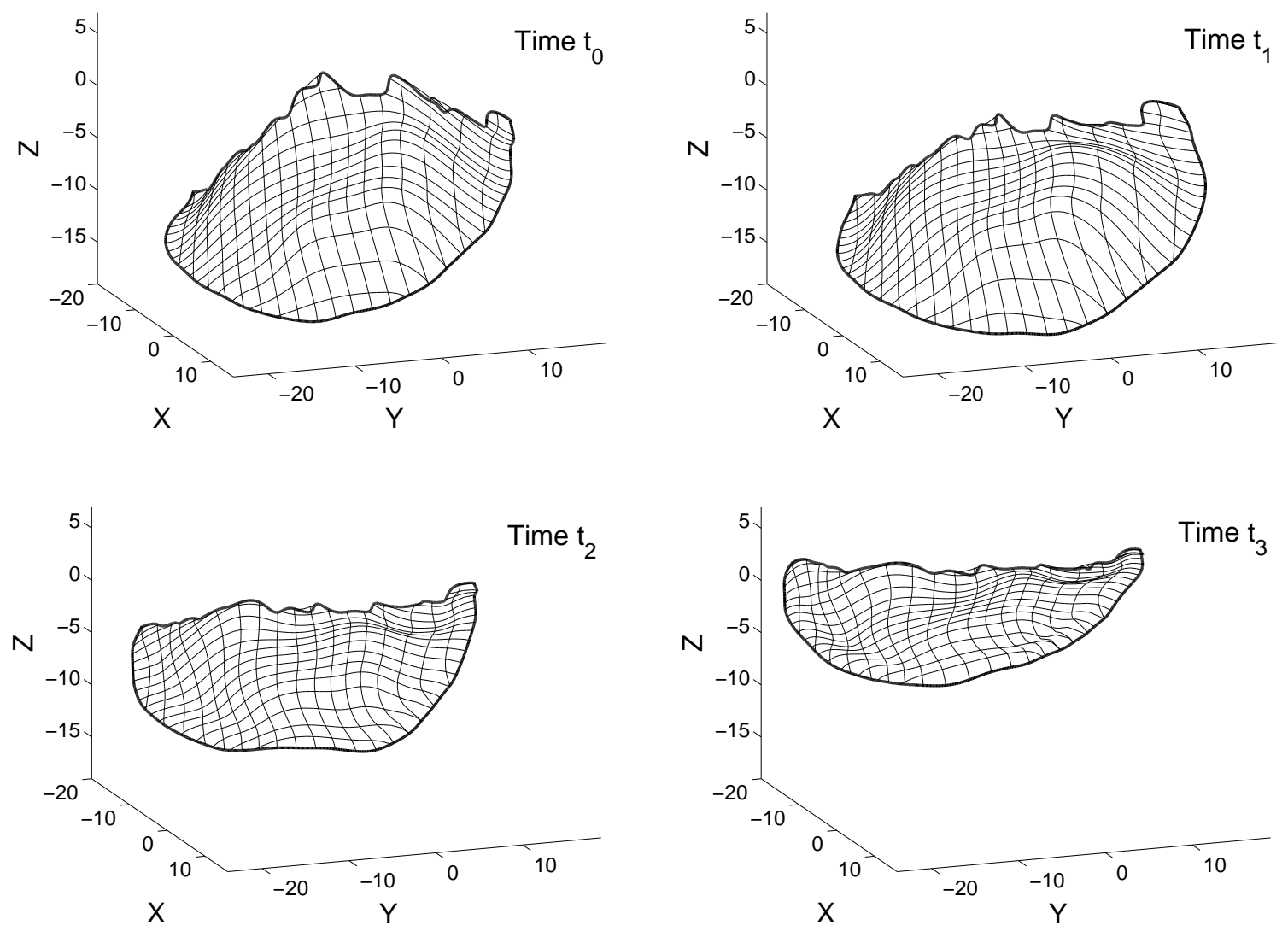

Fig. 6.16. Computed deformations matching four snapshots of the posterior leaflet at instants $0,1,5,10$ 

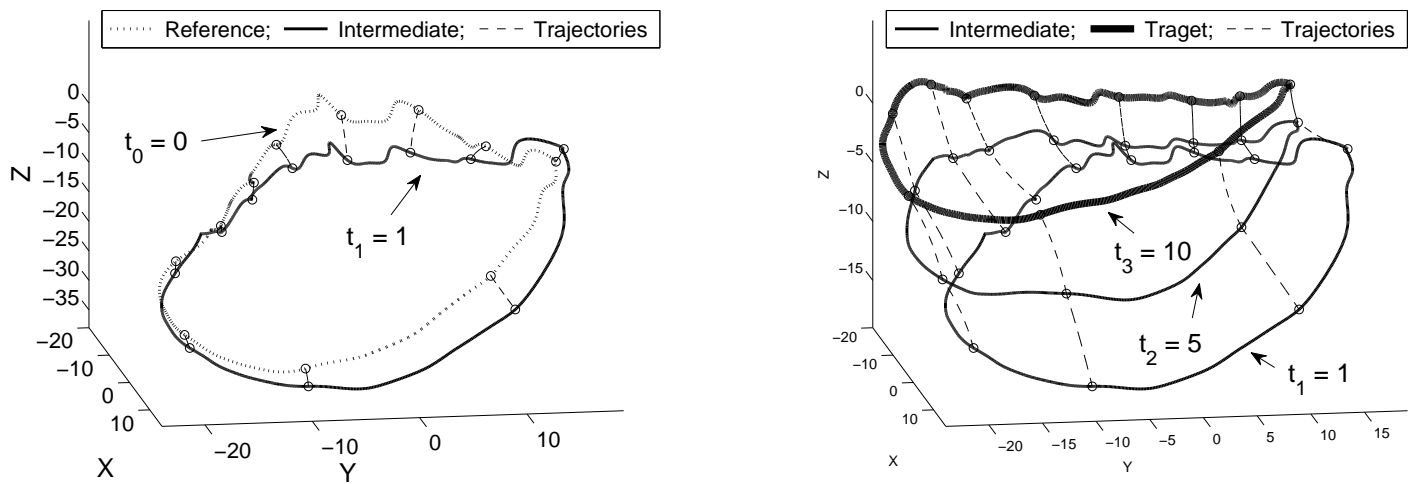

Fig. 6.17. Computed deformations of the posterior leaflet boundary: the continuous boundary deformations are displayed separately for instants 0,1 and for instants $1,5,10$
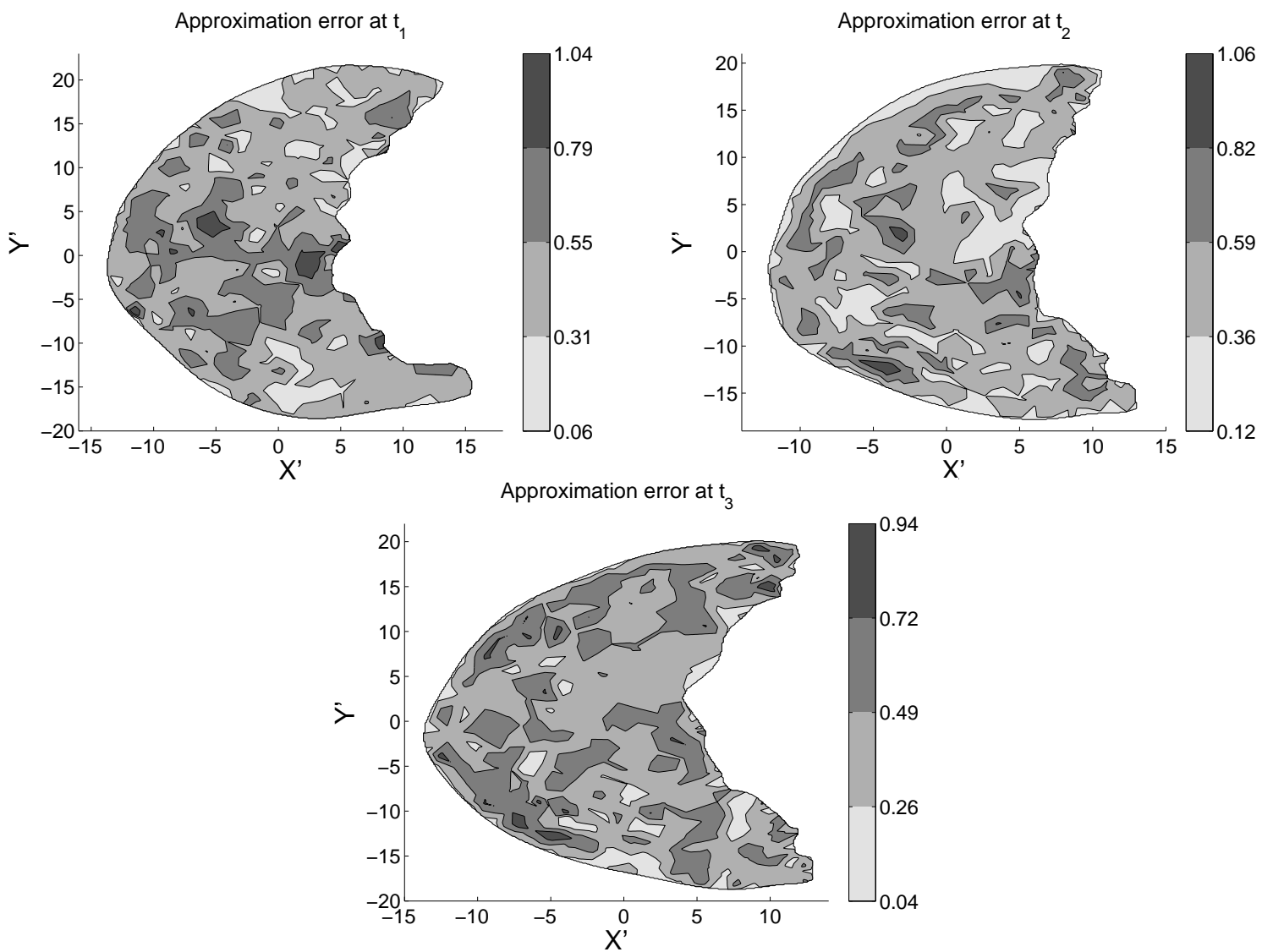

Fig. 6.18. Matching errors between the computed posterior leaflet deformations $\hat{S}_{j}$ and the snapshots $S_{j}$ 


\subsection{Diffeomorphic Matching of the Whole Mitral Valve Apparatus}

We now compute diffeomorphic deformations of the whole mitral valve apparatus ("MVA") viewed as one single composite deformable object as introduced above. We apply the diffeomorphic matching algorithms outlined above to 3 given MVA snapshots $M V A_{0}, M V A_{1}$, $M V A_{2}$, acquired at instants 1,5,10. Our discretization meshes involve roughly 150 points each for the initial anterior leaflet $A L_{0}$ and posterior leaflet $P L_{0}$, and respectively 3200 and 1700 points each for the anterior and posterior leaflets snapshots $A L_{1}, P L_{1}, A L_{2}, P L_{2}$. The smoothed Hausdorff disparities involve several disparity terms for each one of the given MVA snapshots $M V A_{1}$ and $M V A_{2}$, quantifying separately the disparities between AL surfaces, PL surfaces, annulus curves, and coaptation lines. We initialize $\boldsymbol{\alpha}$ by $\boldsymbol{\alpha}=0$. All the choices of parameter values driving the continuation algorithm are identical to the choices made above to compute deformations of the anterior leaflet (see 6.4).
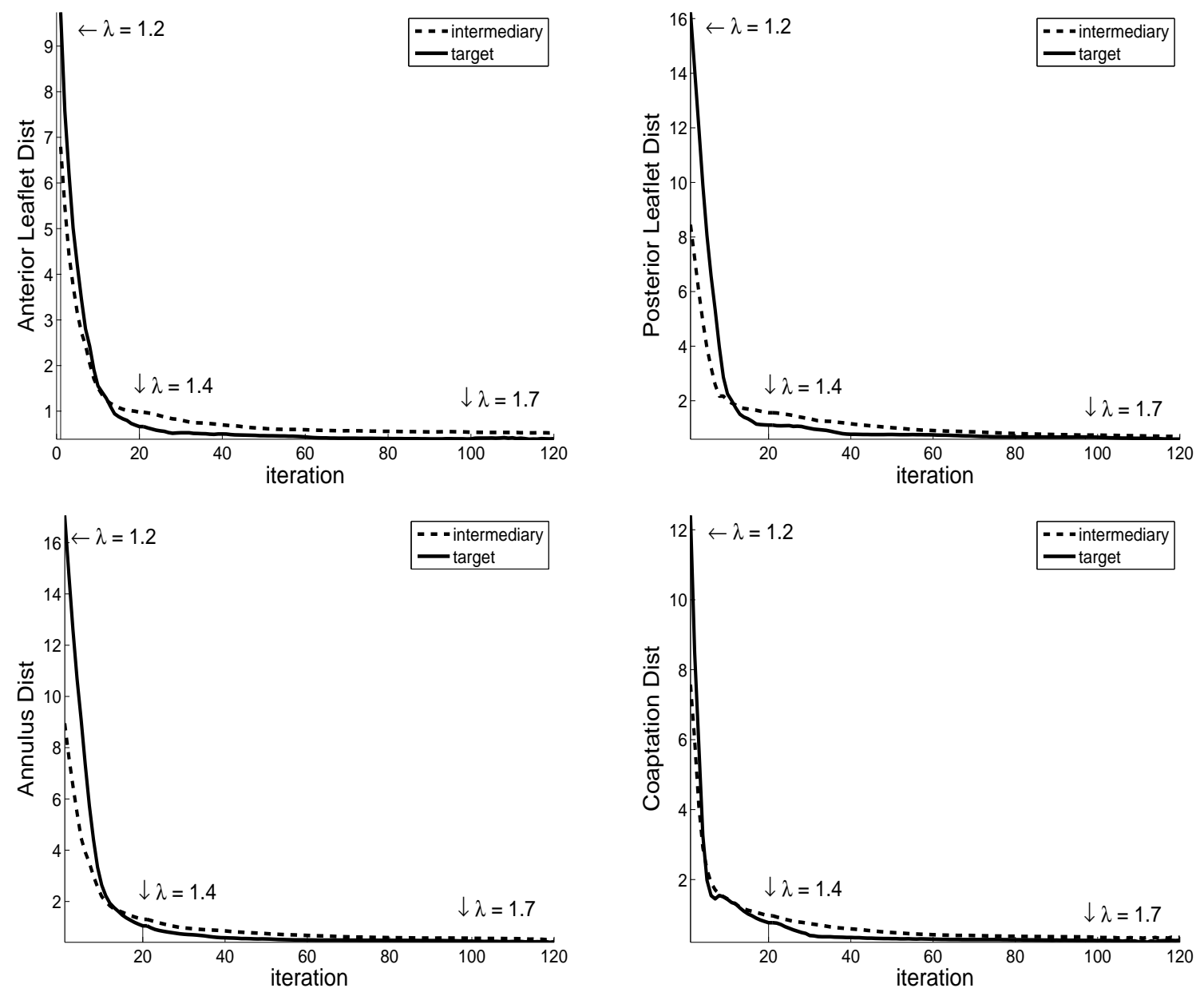

Fig. 6.19. Diffeomorphic matching of the whole Mitral Valve Apparatus: Convergence history for the geometric matching accuracy

Acknowledgments. The work of the authors has been supported by NSF DMS0811153 .

\section{References}

1. N. Arad, N. Dyn, D. Reisfeld, and Y. Yeshurun, Image warping: application to facial expressions, CVGIP: Graphical Models and Image Proc., 56 (1994), pp. 161-172. 
2. L. Armijo, Minimization of functions having Lipschitz continuous first partial derivatives, Pacific J. Math., 16 (1966), pp. 1-3.

3. V.I. Arnold, Sur la géométrie différentielle des groupes de Lie de dimension infinie et ses applications à l'hydrodynamique des fluides parfaits, Ann. Inst. Fourier (Grenoble), 1 (1966), pp. 319-361.

4. N. Aronszajn, Theory of reproducing kernels, Trans. Amer. Math. Soc., 68, 337-404, 1950.

5. R. Azencott, Elastic deformations of soft 3D-shapes, IBIS Colloquium on Medical image analysis. The Methodist Hospital, Kiawah Island, 2006

6. R. Azencott, R. Glowinski, and A. Ramos, A controllability approach to shape identification, Applied Math. Letters 21 (2008), pp. 861-865.

7. R. Azencott, S. Alexander, A. Aggarwaal, A. Jajoo, S. Jain, Y. Li, S. BenZekry (MD), S.H. Little (MD), W. Zoghbi (MD), New parameters to compare Mitral Annulus shapes extracted from 3Dechocardiography, submitted, 2010

8. R. Azencott, A. Jajoo, S. Jain, Y. Li, A. Martynenko,S. BenZekry (MD), S.H. Little (MD), W. Zoghbi (MD), 3D-echocardiographic movies analysis: dynamic deformable models for Mitral Valve, in preparation, 2010

9. M.F. Beg, M.I. Miller, A. Trouvé, and L. Younes, Computing large deformations metric mappings via geodesic flows of diffeomorphisms, Int.J. Comp. Vis., 61(2005), pp 139-157.

10. L. G. Brown, A survey on image registration techniques, ACM Computing Surveys, 24 (1992), pp. $326-376$.

11. Y. Cao, M. I. Miller, R. Winslow, and L. Younes, Large deformation metric mapping of vector fields, IEEE Trans. Med. Imaging, 24 (2005), pp. 1216-1230.

12. A. Chinchuluun, P.M. Pardalos, A. Migdalas, and L. Pitsoulis (eds.), Pareto Optimality, Game Theory and Equilibria, Springer, Berlin-Heidelberg-New York, 2008.

13. H. Chui and A. Rangarajan, A new point matching algorithm for non-rigid registration, Computer Vision and Understanding, 89 (2003), pp. 114-141.

14. M.Chupin, D. Hasboun, S. Baillet, and altri, Competitive segmentation of the hippocampus and volumetry in Alzheimer disease, In: 10th meeting of the Organization for Human Brain Mapping, 2004.

15. J.M. Coron, Control and Nonlinearity, Math. Surveys and Monographs, Vol. 136, American Mathematical Society, Providence, 2007.

16. P. Dupuis, U. Grenander, and M. I. Miller, Variational problems on flows of diffeomorphisms for image matching, Quart. Appl. Math., 56 (1998), pp. 587-600.

17. H. Drury, D.V. Essen, M. Corbetta, and A. Snyder, Surface based analysis of human cerebral cortex, In: Brain Warping, 337-363, Academic Press, 1999.

18. L. Greengard, and J Strain, Fast Gauss transform, SIAM Jour. Sci. Stat. Comp., 12 (1991), pp. 79-94.

19. J. Glaunès, Transport par difféomorphismes de points, de mesures et de courants pour la comparaison des formes et l'anatomie numérique, PhD Thesis, Université Paris 13, 2005.

20. J. Glaunès, A. Qiu, M.I. Miller, and L. Younes, Large deformation diffeomorphic metric curve mapping, Int. J. Comp. Vision, 80 (2008), pp. 317-336.

21. J. Glaunès, A. Trouvé, and L. Younes, Diffeomorphic matching of distributions: A new approach for unlabelled point-sets and sub-manifolds matching, in: 2004 IEEE Computer Society Conference on Computer Vision and Pattern Recognizion (CVPR'04), Vol. 2, IEEE Computer Society, Washington, DC, 2004, pp. $712-718$.

22. J. Glaunès, M. Vaillant, and M. I. Miller, Landmark matching via large deformation diffeomorphisms on the sphere, J. Math. Imaging Vis., 20 (2003), pp. 179-200.

23. R. Glowinski, J.L. Lions, and J. He; Exact and Approximate Controllability for Distributed Parameter Systems: A Numerical Approach, Cambridge University Press, Cambridge, 2008.

24. H. Guo, A. Rangarajan, S. Joshi, and L. Younes, Non-rigid registration of shapes via diffeomorphic point matching, in: Proceedings of the 2004 Int. Symposium on Biomedical Imaging: From Nano to Macro, IEEE Computer Society, Washington, DC, 2004, pp. 924-927.

25. H. Guo, A. Rangarajan, and S. Joshi, Diffeomorphic point matching, in: Handbook of Mathematical Models in Computer Vision, Springer, Berlin-Heidelberg-New York, 2006, pp. 205-219.

26. U. Grenander, and M. I. Miller, Computational anatomy: an emerging discipline, Quart. App. Math., 56 (1998), pp. 617-694.

27. H. Guo, Diffeomorphic point matching with applications in medical image analysis, Ph.D. thesis, Department of Computer and Information Science and Engineering, University of Florida, Gainesville, FL, 2005. 
28. S. Joshi and M. Miller, Landmark matching via large deformation diffeomorphisms, IEEE Trans. Image Processing, 9 (2000), pp. 1357-1370.

29. E. Klassen, A. Srivastava, W. Mio, and S. Joshi, Analysis of planar shapes using geodesic paths on shape spaces, IEEE Trans. Pattern Analysis and Machine Intelligence, 26 (2004), pp. 372-383.

30. J.L. Lions, Controlabilité exacte, perturbation et stabilisation des syst'emes distribués, Masson, Paris, 1988.

31. I. Miller, A,Trouvé, and L.Younes, On the metrics and Euler-Lagrange equations of computational anatomy, Ann. Review of Biomedical Engineering, 4 (2002), pp. 375-405.

32. M. I. Miller and L. Younes, Group action, diffeomorphism and matching: A general framework, Int. J. Comp. Vis., 41 (2001), pp. 61-84.

33. S. Saitoh, Theory of Reproducing Kernels and its Applications, Pitman Research Notes in Mathematics, Vol. 189, Longman, 1988.

34. L. Tartar, Introduction to Sobolev Spaces and Interpolation Theory, Springer, Berlin-Heidelberg-New York, 2007.

35. A. Trouvé, Diffeomorphisms groups and pattern matching in image analysis, Int. J. of Comp. Vis., 28 (1998), pp. 213-221.

36. L. Younes, Computable elastic distances between shapes, SIAM J. Appl. Math. 58 (1998), pp. $565-586$.

37. B. Zitovà and J. Flusser, Image registration methods: a survey, Image and Vision Computing, 21 (2003), pp. 977-1000.

38. E. Zuazua, Controllability and observability of partial differential equations, Chapter 7 in Handbook of Differential Equations, Vol. 3, Evolutionary Equations, Elsevier, Amsterdam, 527-621, (2007). 